\title{
Partner Symmetries, Group Foliation and ASD Ricci-Flat Metrics without Killing Vectors
}

\author{
Mikhail B. SHEFTEL ${ }^{\dagger}$ and Andrei A. MALYKH ${ }^{\ddagger}$ \\ $\dagger$ Department of Physics, Boğaziçi University 34342 Bebek, Istanbul, Turkey \\ E-mail: mikhail.sheftel@boun.edu.tr \\ URL: http://www.phys.boun.edu.tr/faculty_wp/mikhail_sheftel.html \\ $¥$ Department of Numerical Modelling, Russian State Hydrometeorlogical University, \\ 98 Malookhtinsky Ave., 195196 St. Petersburg, Russia \\ E-mail: andrei-malykh@mail.ru
}

Received June 14, 2013, in final form November 19, 2013; Published online November 27, 2013

http://dx.doi.org/10.3842/SIGMA.2013.075

\begin{abstract}
We demonstrate how a combination of our recently developed methods of partner symmetries, symmetry reduction in group parameters and a new version of the group foliation method can produce noninvariant solutions of complex Monge-Ampère equation (CMA) and provide a lift from invariant solutions of CMA satisfying Boyer-Finley equation to non-invariant ones. Applying these methods, we obtain a new noninvariant solution of CMA and the corresponding Ricci-flat anti-self-dual Einstein-Kähler metric with Euclidean signature without Killing vectors, together with Riemannian curvature two-forms. There are no singularities of the metric and curvature in a bounded domain if we avoid very special choices of arbitrary functions of a single variable in our solution. This metric does not describe gravitational instantons because the curvature is not concentrated in a bounded domain.
\end{abstract}

Key words: Monge-Ampère equation; Boyer-Finley equation; partner symmetries; symmetry reduction; non-invariant solutions; group foliation; anti-self-dual gravity; Ricci-flat metric

2010 Mathematics Subject Classification: 35Q75; 83C15

\section{Introduction}

In his pioneer paper [21], Plebañski demonstrated that anti-self-dual (ASD) Ricci-flat metrics on four-dimensional complex manifolds are completely determined by a single scalar potential which satisfies his first or second heavenly equation. Such metrics are solutions to complex vacuum Einstein equations. Real four-dimensional Kähler ASD metrics

$$
d s^{2}=2\left(u_{1 \overline{1}} d z^{1} d \bar{z}^{1}+u_{1 \overline{2}} d z^{1} d \bar{z}^{2}+u_{2 \overline{1}} d z^{2} d \bar{z}^{1}+u_{2 \overline{2}} d z^{2} d \bar{z}^{2}\right)
$$

that solve the vacuum Einstein equations with Euclidean (Riemannian) signature are governed by a scalar real-valued potential $u=u\left(z^{1}, z^{2}, \bar{z}^{1}, \bar{z}^{2}\right)$ which satisfies complex Monge-Ampère equation (CMA)

$$
u_{1 \overline{1}} u_{2 \overline{2}}-u_{1 \overline{2}} u_{2 \overline{1}}=1 \text {. }
$$

A modern justification for this conclusion one can find in the books by Mason and Woodhouse [16] and Dunajski [4]. Here and henceforth, subscripts denote partial derivatives with respect to corresponding variables whereas the bar means complex conjugation, e.g. $u_{1 \overline{1}}=$ $\partial^{2} u / \partial z^{1} \partial \bar{z}^{1}$ and suchlike. The only exception is Section 3 where subscripts of generators $X$ are used to designate different vector fields. 
Among ASD Ricci-flat metrics, the most interesting ones are those that describe gravitational instantons which asymptotically look like a flat space, so that their curvature is concentrated in a finite region of a Riemannian space-time (see [4] and references therein). The most important gravitational instanton is $K 3$ which geometrically is Kummer surface [1], for which an explicit form of the metric is still unknown while many its properties and existence had been discovered and analyzed [8, 28]. A characteristic feature of the $K 3$ instanton is that it does not admit any Killing vectors, that is, no continuous symmetries which implies that the metric potential should be a noninvariant solution of CMA equation. As opposed to the case of invariant solutions, for noninvariant solutions of CMA there should be no symmetry reduction in the number of independent variables. Since standard methods of Lie group analysis of PDEs provide only invariant solutions, which implies symmetry reduction in the solutions and hence in the metric (1.1), they cannot be applied for obtaining noninvariant solutions to the CMA equation and ASD Ricci-flat metrics without Killing vectors. Thus, to obtain at least some pieces of $K 3$ metric explicitly one needs a technique for deriving non-invariant solutions of multi-dimensional non-linear equations.

In the previous papers $[10,11,13]$ we have developed methods for obtaining noninvariant solutions though still remaining in the symmetry framework. We extended the Lax equations of Mason and Newman for CMA [15, 16] by supplementing them with another pair of linear equations, so that CMA becomes an algebraic consequence of these equations, whereas the original Lax pair generated only differential consequences of CMA with no distinction between elliptic, hyperbolic and homogeneous versions of CMA. The equation, determining symmetry characteristics [19] for CMA, now appears as the integrability condition of our linear equations. Moreover, the symmetry condition has a two-dimensional divergence form and therefore uniquely determines locally a potential function which turns out to be a solution to the symmetry condition, that is, the potential of a symmetry is also a symmetry, which is a characteristic feature of a more general class of Monge-Ampère equations [25]. We called this pair of symmetries, the original one and the corresponding potential, partner symmetries and applied them to generate noninvariant solutions of CMA and corresponding heavenly metrics without Killing vectors. We discovered that the equations connecting partner symmetries can be treated as an invariance condition for solutions of CMA with respect to a certain nonlocal symmetry constructed from partner symmetries and nonlocal recursion operators [10]. This was close but not identical to the idea of hidden symmetries by Dunajski and Mason [6]. The invariance of solutions under the nonlocal symmetry, unlike the invariance under a local symmetry, does not imply the symmetry reduction in the number of independent variables, so such solutions are noninvariant in the usual sense.

The problem of finding solutions of CMA by solving the equations for partner symmetries was facilitated by the observation that the full system of such equations provides a lift from invariant solutions of CMA to its noninvariant solutions [12, 13, 24]. As is shown in [2], any three dimensional reduction of CMA leads to either translationally invariant solutions satisfying 3-dimensional Laplace equation or to rotationally invariant solutions satisfying elliptic BoyerFinley (BF) equation. Earlier, in [13], we showed that there exists a lift of translationally invariant solutions of CMA to noninvariant solutions. In [12] we obtained a lift from hyperbolic version of Boyer-Finley equation to noninvariant solutions of the hyperbolic CMA, with 1 replaced by -1 on the right-hand side of (1.2). In this paper we will show that there also exist rotationally invariant solutions, related to solutions of elliptic Boyer-Finley equation, which can be lifted to noninvariant solutions of the elliptic CMA (1.2). Therefore, one may start with a simpler problem of finding translationally or rotationally invariant solutions and then, using the partner symmetries, lift them to noninvariant solutions.

Another modification of the method of partner symmetries was, by using Lie equations, to introduce explicitly symmetry group parameters (for commuting symmetries) as additional independent variables of the problem instead of symmetry characteristics [13]. This trick allowed 
us to discover an integrability condition of the equations for partner symmetries. Furthermore, this made possible using symmetry reductions in group parameters without symmetry reductions in "physical variables" in order to simplify the equations to be solved without ending up with invariant solutions.

In this paper, we apply to the full system of equations for partner symmetries of CMA another solution tool, group foliation [14, 18, 23], which we used before for CMA and the Boyer-Finley equation [2]. The idea of the group foliation belongs to S. Lie [9] and was developed by E. Vessiot [27] and a modern presentation was given by L.V. Ovsiannikov [20]. Original differential equations are foliated with respect to a chosen symmetry (sub)group into automorphic equations describing orbits of the symmetry group and resolving equations determining a collection of the orbits. The automorphic property of the first subsystem of equations means that any of its solutions can be obtained from any other solution by some transformation of the chosen symmetry subgroup. This property makes the automorphic system completely integrable if only one of its solutions can be obtained. Thus, the problem reduces to obtaining as many particular solutions of the resolving system as possible. Each solution will fix a particular automorphic system and the corresponding orbit in the solution space of original equations.

Applying this method to equations of partner symmetries, we find a very large number of resolving equations for which it is extremely difficult to find even a single solution. Therefore, we use our modification of the method which utilizes to a greater degree operators of invariant differentiation. They are defined by the property that they commute with any prolongation of the symmetry generators of the Lie group chosen for the foliation and hence they map differential invariants again into differential invariants. Our discovery was that the resolving system is equivalent to the commutator algebra of operators of invariant differentiation together with its Jacobi identities, so that we can replace the problem of solving the resolving equations by the problem of solving commutator relations between the operators of invariant differentiation. An example of such approach was given in our papers [14, 18, 23].

In Section 2, we derive the extended system of six equations for partner symmetries of CMA including their integrability condition and introduce symmetry group parameters as additional independent variables.

In Section 3, we determine all point symmetries of the extended system and perform its symmetry reduction with respect to two group parameters. This is not a symmetry reduction with respect to "physical" variables and so it does not imply a symmetry reduction of solutions of CMA. Our aim here is to prepare the ground for a lift of some solutions to the elliptic version of the Boyer-Finley (BF) equation to noninvariant solutions of CMA. We determine all point symmetries of the reduced system. To have BF in our system, we choose the symmetry of simultaneous rotations in complex $z^{1}$ - and $z^{2}$-planes for a further reduction which picks up rotationally invariant solutions of CMA. Being followed by a Legendre transformation, this yields the BF equation. Meanwhile, we also keep non-reduced transformed CMA equation obtained from the integrability condition of the extended system, though in different variables involving symmetry group parameters. If we find a solution to BF which also satisfies all other equations of the extended system, this would mean a lift from rotationally invariant solutions to noninvariant solutions of CMA. Finally, we determine all point symmetries of the transformed extended system which we will need for the group foliation.

In Section 4, we choose a complex conjugate pair of the symmetry generators which contain maximum number of arbitrary functions and thus generate a maximal infinite symmetry subgroup for the group foliation. We determine all first-order operators of invariant differentiation and obtain a set of all differential invariants up to the second order, inclusive.

In Section 5, we perform the group foliation deriving the full set of automorphic and resolving equations. We derive also the commutator algebra of operators of invariant differentiation. There are too many resolving equations for a straightforward search of its particular solutions. 
In Section 6, we find some solutions of the extended system by applying our strategy of making ansatzes, that simplify the commutator algebra of operators of invariant differentiation, rather than trying to solve directly a huge system of the resolving equations. As a by-product, we obtain new solutions of the Boyer-Finley equation. We choose more generally looking solution for lifting it to a solution of CMA.

In Section 7, we apply Legendre transformations of our solution to a solution of CMA equation with a parameter-dependent right-hand side. This solution depends on three arbitrary functions of a single complex variable together with their complex conjugates. This is also a simultaneous solution of the transformed Boyer-Finley equation in somewhat different variables which is related to a symmetry reduction of CMA and hence determines its invariant solutions. Therefore, our construction provides a lift from invariant to non-invariant solutions of CMA.

In Section 8, we use our solution of the CMA equation to obtain Kähler metric of Euclidean signature. This metric is anti-self-dual and Ricci-flat and have pole singularities in a bounded domain only for a special choice of arbitrary functions in our solution. By avoiding this special choice we obtain the metric without such singularities. We have also computed Riemannian curvature two-forms which do not depend on two variables and hence the curvature does not vanish outside of a bounded domain in the space of all variables. This means that our metric does not describe a gravitational instanton. Even though this simplest possible example of application of our approach does not produce an instanton metric, we believe that more refined solutions to our extended system of equations for partner symmetries and/or different chain of reductions will yield a gravitational instanton metric with no Killing vectors.

In Section 9, we derive the invariance conditions for our solution under the symmetries of the parameter-dependent CMA. A detailed study of these conditions proves that generically (without special restrictions on arbitrary functions in our solution to CMA) the solution is noninvariant and hence the corresponding metric has no Killing vectors.

\section{Basic equations}

In this section, we derive a complete set of equations for partner symmetries to provide a tool for obtaining noninvariant solutions of CMA while remaining in the symmetry group framework $[10,11]$. Here we introduce symmetry group parameters as additional independent variables in order to reserve a possibility of symmetry reduction in group parameters to simplify these equations without reduction in the number of original independent variables in CMA and thus avoiding ending up with invariant solutions. Using these additional variables also facilitates a derivation of the integrability condition for our equations, so that the extended set of equations augmented with this integrability condition is integrable in the sense of Frobenius: all further integrability conditions are direct algebraic or differential consequences of already available equations.

For the complex Monge-Ampère equation (1.2) the symmetry condition, that determines symmetry characteristics $\varphi$ of (1.2),

$$
u_{1 \overline{1}} \varphi_{2 \overline{2}}+u_{2 \overline{2}} \varphi_{1 \overline{1}}-u_{1 \overline{2}} \varphi_{2 \overline{1}}-u_{2 \overline{1}} \varphi_{1 \overline{2}}=0
$$

on solutions of CMA. Here the subscripts of $\varphi$ denote total derivatives with respect to corresponding independent variables. Equation (2.1) can be set in the total divergence form

$$
\left(u_{1 \overline{1}} \varphi_{2}-u_{2 \overline{1}} \varphi_{1}\right)_{\overline{2}}-\left(u_{1 \overline{2}} \varphi_{2}-u_{2 \overline{2}} \varphi_{1}\right)_{\overline{1}}=0 .
$$

We assume that all functions that we operate with are smooth, that is, they have continuous derivatives of any required order. Then equation (2.2) suggests local existence of potential $\psi$ 
defined by the equations

$$
\psi_{\overline{1}}=u_{1 \overline{1}} \varphi_{2}-u_{2 \overline{1}} \varphi_{1}, \quad \psi_{\overline{2}}=u_{1 \overline{2}} \varphi_{2}-u_{2 \overline{2}} \varphi_{1}
$$

in the sense that the condition (2.2) becomes just the equality of mixed derivatives $\left(\psi_{\overline{1}}\right)_{\overline{2}}=\left(\psi_{\overline{2}}\right)_{\overline{1}}$, together with the complex conjugate equations

$$
\bar{\psi}_{1}=u_{1 \overline{1}} \bar{\varphi}_{\overline{2}}-u_{1 \overline{2}} \bar{\varphi}_{\overline{1}}, \quad \bar{\psi}_{2}=u_{2 \overline{1}} \bar{\varphi}_{\overline{2}}-u_{2 \overline{2}} \bar{\varphi}_{\overline{1}} .
$$

We do not discuss here the difficult problem of the global existence of potential $\psi$.

A straightforward check shows that the potential $\psi$ also satisfies symmetry condition (2.1), so that $\psi$ is also a symmetry if $\varphi$ is a symmetry and hence the relations (2.3) and (2.4) are recursion relations for symmetries ("partner symmetries"). Transformation (2.3) is algebraically invertible since its determinant equals one due to (1.2). Inverse transformation has the form

$$
\varphi_{1}=u_{1 \overline{2}} \psi_{\overline{1}}-u_{1 \overline{1}} \psi_{\overline{2}}, \quad \varphi_{2}=u_{2 \overline{2}} \psi_{\overline{1}}-u_{2 \overline{1}} \psi_{\overline{2}}
$$

together with its complex conjugate

$$
\bar{\varphi}_{\overline{1}}=u_{2 \overline{1}} \bar{\psi}_{1}-u_{1 \overline{1}} \bar{\psi}_{2}, \quad \bar{\varphi}_{\overline{2}}=u_{2 \overline{2}} \bar{\psi}_{1}-u_{1 \overline{2}} \bar{\psi}_{2} .
$$

For symmetries with characteristics $\varphi, \bar{\varphi}, \psi$ and $\bar{\psi}$, Lie equations read

$$
\varphi=u_{\tau}, \quad \bar{\varphi}=u_{\bar{\tau}}, \quad \psi=u_{\sigma}, \quad \bar{\psi}=u_{\bar{\sigma}},
$$

where $\tau, \sigma$ together with their complex conjugates $\bar{\tau}, \bar{\sigma}$ are group parameters. Simultaneous inclusion of several group parameters as additional independent variables implies the commutativity conditions for corresponding symmetries in the form

$$
\varphi_{\bar{\tau}}=\bar{\varphi}_{\tau}, \quad \psi_{\bar{\sigma}}=\bar{\psi}_{\sigma}, \quad \varphi_{\sigma}=\psi_{\tau}, \quad \varphi_{\bar{\sigma}}=\bar{\psi}_{\tau}
$$

and complex conjugates to the last two equations.

We now use (2.7) to replace symmetry characteristics by derivatives of $u$ with respect to group parameters in equations $(2.3),(2.5)$ and their complex conjugates $(2.4),(2.6)$ with the result

$$
\begin{array}{ll}
u_{\sigma \overline{1}}=u_{1 \overline{1}} u_{\tau 2}-u_{2 \overline{1}} u_{\tau 1}, & u_{\sigma \overline{2}}=u_{1 \overline{2}} u_{\tau 2}-u_{2 \overline{2}} u_{\tau 1}, \\
u_{\tau 1}=u_{1 \overline{2}} u_{\sigma \overline{1}}-u_{1 \overline{1}} u_{\sigma \overline{2}}, & u_{\tau 2}=u_{2 \overline{2}} u_{\sigma \overline{1}}-u_{2 \overline{1}} u_{\sigma \overline{2}},
\end{array}
$$

and the complex conjugate equations

$$
\begin{array}{ll}
u_{\bar{\sigma} 1}=u_{1 \overline{1}} u_{\bar{\tau} \overline{2}}-u_{\overline{2} 1} u_{\bar{\tau} \overline{1}}, & u_{\bar{\sigma} 2}=u_{\overline{1} 2} u_{\bar{\tau} \overline{2}}-u_{2 \overline{2}} u_{\bar{\tau} \overline{1}}, \\
u_{\bar{\tau} \overline{1}}=u_{\overline{1} 2} u_{\bar{\sigma} 1}-u_{1 \overline{1}} u_{\bar{\sigma} 2}, & u_{\bar{\tau} \overline{2}}=u_{2 \overline{2}} u_{\bar{\sigma} 1}-u_{\overline{2} 1} u_{\bar{\sigma} 2} .
\end{array}
$$

We note that four equations (2.9) and (2.11) are algebraic consequences of other equations (2.8), (2.10) and CMA. We note also that CMA itself follows as an algebraic consequence from equations (2.8), (2.10) and the first equation in (2.11).

To study integrability conditions of our system, we set the first equations in (2.8) and (2.11) in the form

$$
\left(u_{1 \overline{1}} u_{2}\right)_{\tau}=\left(u_{\sigma}+u_{2} u_{\tau 1}\right)_{\overline{1}}, \quad\left(u_{1 \overline{1}} u_{2}\right)_{\bar{\sigma}}=\left(u_{2} u_{\bar{\sigma} 1}-u_{\bar{\tau}}\right)_{\overline{1}} .
$$

Equations (2.12) constitute an active system since they have a second-order nontrivial integrability condition obtained by cross differentiation of these equations with respect to $\bar{\sigma}$ and $\tau$ and further integration with respect to $\bar{z}^{1}$

$$
u_{\tau \bar{\tau}}+u_{\sigma \bar{\sigma}}+u_{\bar{\sigma} 2} u_{\tau 1}-u_{\bar{\sigma} 1} u_{\tau 2}=0,
$$


where the "constant" of integration can be eliminated by a redefinition of $u$. To make this equation self-conjugate, we multiply it with an overall factor $u_{1 \overline{1}}$ and then eliminate $u_{1 \overline{1}} u_{\bar{\sigma} 2}$ and $u_{1 \overline{1}} u_{\tau 2}$ in the last two terms of (2.12) using first equations in (2.11) and (2.8), respectively, with the final form of the integrability condition

$$
u_{1 \overline{1}}\left(u_{\tau \bar{\tau}}+u_{\sigma \bar{\sigma}}\right)-u_{\tau 1} u_{\bar{\tau} \overline{1}}-u_{\sigma \overline{1}} u_{\bar{\sigma} 1}=0 .
$$

In a similar way, we obtain the alternative form of the integrability condition

$$
u_{2 \overline{2}}\left(u_{\tau \bar{\tau}}+u_{\sigma \bar{\sigma}}\right)-u_{\tau 2} u_{\bar{\tau} \overline{2}}-u_{\sigma \overline{2}} u_{\bar{\sigma} 2}=0 .
$$

We can choose (2.8), (2.10), (2.13) and CMA for the set of algebraically independent equations. All other equations are linearly dependent on the chosen equations. One could also check that there are no further independent second-order integrability conditions of our system of six equations.

\section{Reduction of partner symmetries system for CMA}

Here we study point symmetries of the extended system for partner symmetries and use two symmetry reductions with respect to group parameters to simplify this system. The choice of the first reduction obeys the requirement for the reduced integrability condition (3.6) to be related to CMA (3.7) in new variables. The second symmetry reduction of the extended system results in rotational reduction of the original CMA (1.2) which yields equation (3.9) related to the Boyer-Finley equation (3.18) by Legendre transformation (3.15) combined with the differential substitution $w=v_{t}$ and the following integration of equations with respect to $t$. The transformed reduced integrability condition (3.12) becomes the Legendre transform of the Monge-Ampère equation (3.13). In this way we arrive at the system which contains both CMA and Boyer-Finley equation (BF) thus providing the possibility of a lift from rotationally invariant solutions of CMA (related to solutions of BF) to noninvariant solutions of CMA. The Legendre transformation appears as a necessary step because of the well-known relation between the rotational reduction of CMA and BF equations (see, e.g., (4.10), (4.12) in [2]).

We list the generators of all point symmetries of the extended system of six equations CMA, (2.8), (2.10) and (2.13)

$$
\begin{aligned}
& X_{1}=\partial_{\tau}, \quad \bar{X}_{1}=\partial_{\bar{\tau}}, \quad X_{2}=\partial_{\sigma}, \quad \bar{X}_{2}=\partial_{\bar{\sigma}}, \quad X_{3}=\tau \partial_{\tau}+\sigma \partial_{\sigma}, \\
& \bar{X}_{3}=\bar{\tau} \partial_{\bar{\tau}}+\bar{\sigma} \partial_{\bar{\sigma}}, \quad X_{4}=z^{2} \partial_{2}-\bar{z}^{2} \partial_{\overline{2}}+\bar{\tau} \partial_{\bar{\tau}}-\tau \partial_{\tau}+\sigma \partial_{\sigma}-\bar{\sigma} \partial_{\bar{\sigma}}, \\
& X_{5}=\tau \partial_{\bar{\sigma}}-\sigma \partial_{\bar{\tau}}, \quad \bar{X}_{5}=\bar{\tau} \partial_{\sigma}-\bar{\sigma} \partial_{\tau} \quad X_{6}=z^{2} \partial_{2}+\bar{z}^{2} \partial_{\overline{2}}+u \partial_{u}, \\
& X_{a}=a\left(z^{1}, z^{2}, \bar{\tau}, \sigma\right) \partial_{u}, \quad X_{\bar{a}}=\bar{a}\left(\bar{z}^{1}, \bar{z}^{2}, \tau, \bar{\sigma}\right) \partial_{u}, \\
& X_{c}=c_{z^{1}} \partial_{2}-c_{z^{2}} \partial_{1}+\left(\tau c_{\sigma}-\bar{\sigma} c_{\bar{\tau}}\right) \partial_{u}, \\
& X_{\bar{c}}=\bar{c}_{\bar{z}^{1}} \partial_{\overline{2}}-\bar{c}_{\bar{z}^{2}} \partial_{\overline{1}}+\left(\bar{\tau} \bar{c}_{\bar{\sigma}}-\sigma \bar{c}_{\tau}\right) \partial_{u}, \quad X_{f}=f(\tau, \sigma, \bar{\tau}, \bar{\sigma}) \partial_{u},
\end{aligned}
$$

where $a, \bar{a}, c=c\left(z^{1}, z^{2}, \bar{\tau}, \sigma\right), \bar{c}=\bar{c}\left(\bar{z}^{1}, \bar{z}^{2}, \tau, \bar{\sigma}\right)$ are arbitrary functions and $f(\tau, \sigma, \bar{\tau}, \bar{\sigma})$ satisfies the equation $f_{\tau \bar{\tau}}+f_{\sigma \bar{\sigma}}=0$. We note that obvious translational symmetry generators $\partial_{1}$, $\partial_{\overline{1}}, \partial_{2}$ and $\partial_{\overline{2}}$ are particular cases of the generators $X_{c}$ and $X_{\bar{c}}$. We have to emphasize that the subscripts of $X$ designate different vector fields, contrary to our previous convention that subscripts denote partial derivatives.

We specify two symmetries from (3.1) for a symmetry reduction of the extended system

$$
X_{I}=\partial_{\tau}-\partial_{1}, \quad \bar{X}_{I}=\partial_{\bar{\tau}}-\partial_{\overline{1}} .
$$


Solutions of CMA invariant with respect to symmetries (3.2) are determined by the conditions

$$
u_{\tau}=u_{1}, \quad u_{\bar{\tau}}=u_{\overline{1}}
$$

Using (3.3), we eliminate $u_{\tau}$ and $u_{\bar{\tau}}$ in all the equations (2.8), (2.10) and (2.13) to obtain

$$
\begin{aligned}
& u_{\sigma \overline{1}}=u_{1 \overline{1}} u_{12}-u_{2 \overline{1}} u_{11}, \quad u_{\sigma \overline{2}}=u_{1 \overline{2}} u_{12}-u_{2 \overline{2}} u_{11}, \\
& u_{\bar{\sigma} 1}=u_{1 \overline{1}} u_{\overline{1} \overline{2}}-u_{\overline{2} 1} u_{\overline{1} \overline{1}}, \quad u_{\bar{\sigma} 2}=u_{\overline{1} 2} u_{\overline{1} \overline{2}}-u_{2 \overline{2}} u_{\overline{1} \overline{1}}, \\
& u_{1 \overline{1}} u_{\sigma \bar{\sigma}}-u_{1 \bar{\sigma}} u_{\overline{1} \sigma}=u_{11} u_{\overline{1} \overline{1}}-u_{1 \overline{1}}^{2} .
\end{aligned}
$$

We note that equation (3.6) can be obtained by the Legendre transformation

$$
v=u-z^{1} u_{1}-\bar{z}^{1} u_{\overline{1}}, \quad p=-u_{1}, \quad \bar{p}=-u_{\overline{1}}
$$

of the CMA in new variables

$$
v_{p \bar{p}} v_{\sigma \bar{\sigma}}-v_{p \bar{\sigma}} v_{\sigma \bar{p}}=1
$$

All point symmetry generators of the system of equations CMA, (3.4), (3.5) and (3.6) are listed below

$$
\begin{aligned}
& X_{1}=z^{1} \partial_{1}-\bar{z}^{1} \partial_{\overline{1}}-2\left(z^{2} \partial_{2}-\bar{z}^{2} \partial_{\overline{2}}\right), \quad X_{2}=z^{2} \partial_{2}+\bar{z}^{2} \partial_{\overline{2}}+u \partial_{u}, \\
& X_{3}=a(\sigma) \partial_{2}+\frac{1}{2 \lambda}\left(z^{1}\right)^{2} a^{\prime}(\sigma) \partial_{u}, \quad X_{4}=b(\bar{\sigma}) \partial_{\overline{2}}+\frac{\lambda}{2}\left(\bar{z}^{1}\right)^{2} b^{\prime}(\bar{\sigma}) \partial_{u}, \\
& X_{5}=c^{\prime}(\sigma)\left(z^{1} \partial_{1}-z^{2} \partial_{2}\right)+c(\sigma) \partial_{\sigma}-\frac{1}{2 \lambda}\left(z^{1}\right)^{2} z^{2} c^{\prime \prime}(\sigma) \partial_{u}, \\
& X_{6}=d^{\prime}(\bar{\sigma})\left(\bar{z}^{1} \partial_{\overline{1}}-\bar{z}^{2} \partial_{\overline{2}}\right)+d(\bar{\sigma}) \partial_{\bar{\sigma}}-\frac{\lambda}{2}\left(\bar{z}^{1}\right)^{2} \bar{z}^{2} d^{\prime \prime}(\bar{\sigma}) \partial_{u}, \\
& X_{7}=-\lambda f_{z^{2}}\left(z^{2}, \sigma\right) \partial_{1}+z^{1} f_{\sigma}\left(z^{2}, \sigma\right) \partial_{u} \\
& X_{8}=-\frac{1}{\lambda} g_{\bar{z}^{2}}\left(\bar{z}^{2}, \bar{\sigma}\right) \partial_{\overline{1}}+\bar{z}^{1} g_{\bar{\sigma}}\left(\bar{z}^{2}, \bar{\sigma}\right) \partial_{u}, \\
& X_{9}=h\left(z^{2}, \sigma\right) \partial_{u}, \quad X_{10}=k\left(\bar{z}^{2}, \bar{\sigma}\right) \partial_{u} .
\end{aligned}
$$

To arrive at the Boyer-Finley equation, we need rotationally invariant solutions of CMA [2]. Among the symmetries (3.8) of our extended system we can choose the symmetry of simultaneous rotations in $z^{1}$ and $z^{2}$ complex planes, generated by $X_{1}$, which is convenient to combine with the point transformation $z^{2}=e^{p}, \bar{z}^{2}=e^{\bar{p}}$. The symmetry generator becomes $X=-i X_{1}=$ $2 i\left(\partial_{p}-\partial_{\bar{p}}\right)-i\left(z^{1} \partial_{1}-\bar{z}^{1} \partial_{\overline{1}}\right)$, so that new invariant variables are $\rho=p+\bar{p}, q=z^{1} e^{p / 2}, \bar{q}=\bar{z}^{1} e^{\bar{p} / 2}$ and $u=u(\rho, q, \bar{q}, \sigma, \bar{\sigma})$.

After this symmetry reduction the equations CMA, (3.4), (3.5) and (3.6) become respectively

$$
\begin{aligned}
& u_{q \bar{q}} u_{\rho \rho}-u_{\rho q} u_{\rho \bar{q}}=e^{\rho / 2}, \\
& u_{\sigma \bar{q}}=u_{q \bar{q}}\left(u_{\rho q}+u_{q} / 2\right)-u_{q q} u_{\rho \bar{q}}, \\
& u_{\sigma \rho}=u_{\rho q}\left(u_{\rho q}+u_{q} / 2\right)-u_{q q} u_{\rho \rho}
\end{aligned}
$$

together with complex conjugates of (3.10) and (3.11) and, finally,

$$
u_{q \bar{q}} u_{\sigma \bar{\sigma}}-u_{\sigma \bar{q}} u_{\bar{\sigma} q}=e^{\rho / 2}\left(u_{q q} u_{\bar{q} \bar{q}}-u_{q \bar{q}}^{2}\right) .
$$

We note that equation (3.12) has the form of the Legendre transform (3.6) of the Monge-Ampère equation in variables $q, \bar{q}, \sigma, \bar{\sigma}$ with $\rho$ playing the role of a parameter, similarly to our remark 
after equation (3.6). Parameter $\rho$ can be scaled away by changing $\sigma, \bar{\sigma}$ to the new variables $s, \bar{s}$ defined by $s=\sigma e^{\rho / 4}, \bar{s}=\bar{\sigma} e^{\rho / 4}$ when the equation (3.12) becomes

$$
u_{q \bar{q}} u_{s \bar{s}}-u_{s \bar{q}} u_{\bar{s} q}=u_{q q} u_{\bar{q} \bar{q}}-u_{q \bar{q}}^{2}
$$

which is exactly the Monge-Ampère equation

$$
v_{p \bar{p}} v_{s \bar{s}}-v_{p \bar{s}} v_{s \bar{p}}=1
$$

after the Legendre transformation

$$
v=u-q u_{q}-\bar{q} u_{\bar{q}}, \quad p=-u_{q}, \quad \bar{p}=-u_{\bar{q}} .
$$

Therefore, our system contains also transformed Monge-Ampère equation (3.13), though in different variables, in the form (3.12).

Our aim is to transform the reduced CMA to the Boyer-Finley equation. On the way to it, we apply one-dimensional Legendre transformation

$$
t=u_{\rho}, \quad w=u-\rho u_{\rho}, \quad \rho=-w_{t}, \quad u=w-t w_{t} .
$$

CMA equation (3.9) becomes

$$
w_{q \bar{q}}=-w_{t t} e^{-w_{t} / 2} .
$$

Equations (3.10) and (3.11) take the form

$$
w_{\sigma \bar{q}}=\frac{1}{2} w_{q} w_{q \bar{q}}+w_{t q} e^{-w_{t} / 2}, \quad w_{t \sigma}=\frac{1}{2} w_{q} w_{t q}-w_{q q}
$$

and complex conjugate equations. The image of equation (3.12) with the use of other equations becomes

$$
w_{\sigma \bar{\sigma}}=w_{t t} e^{-w_{t}}+\frac{1}{2}\left(w_{\bar{q}} w_{t q}+w_{q} w_{t \bar{q}}-\frac{1}{2} w_{q} w_{\bar{q}} w_{t t}\right) e^{-w_{t} / 2} .
$$

The final step is to set $w=v_{t}$, which makes all the equations to be total derivatives with respect to $t$, and then integrate the equations with respect to $t$. To simplify the notation, we also change $\sigma$ to $z$ and $\bar{\sigma}$ to $\bar{z}$. The reduced CMA takes the form of the Boyer-Finley equation

$$
v_{q \bar{q}}=2 e^{-v_{t t} / 2} .
$$

Equations (3.16) together with their complex conjugates read

$$
\begin{aligned}
v_{q q} & =-v_{t z}+\frac{1}{4} v_{t q}^{2}, \\
v_{\bar{q} \bar{q}} & =-v_{t \bar{z}}+\frac{1}{4} v_{t \bar{q}}^{2}, \\
v_{\bar{q} z} & =v_{t q} e^{-v_{t t} / 2}, \\
v_{q \bar{z}} & =v_{t \bar{q}} e^{-v_{t t} / 2},
\end{aligned}
$$

and the equation (3.17) becomes

$$
v_{z \bar{z}}=-e^{-v_{t t}}+\frac{1}{2} v_{t q} v_{t \bar{q}} e^{-v_{t t} / 2}
$$


According to our remark after equation (3.14), our final system contains besides Boyer-Finley equation (3.18) also transformed Monge-Ampère equation, which is a consequence of this system, though its explicit form, being a bit lengthy, is not presented here. Therefore, we have hopes that finding some noninvariant solutions of the Boyer-Finley equation, we will be able to lift them to noninvariant solutions of the complex Monge-Ampère equation, this being our main goal.

Point symmetries generators of the above system are

$$
\begin{aligned}
& X_{1}=\partial_{t}, \quad X_{2}=q \partial_{q}+\bar{q} \partial_{\bar{q}}+2 t \partial_{t}+\left(4 v-2 t^{2}\right) \partial_{v}, \quad X_{3}=q a(z) \partial_{v}, \quad X_{4}=\bar{X}_{3}, \\
& X_{5}=b(z) \partial_{v}, \quad X_{6}=\bar{X}_{5}, \quad X_{7}=\left(t c(z)-q^{2} c^{\prime}(z) / 2\right) \partial_{v}, \quad X_{8}=\bar{X}_{7}, \\
& X_{9}=d(z) \partial_{q}+\left\{q^{3} d^{\prime \prime}(z) / 3-2 q t d^{\prime}(z)\right\} \partial_{v}, \quad X_{10}=\bar{X}_{9}, \\
& X_{11}=\frac{1}{2} f^{\prime}(z) q \partial_{q}+f(z) \partial_{z}+\left\{q^{4} f^{\prime \prime \prime}(z) / 24-t q^{2} f^{\prime \prime}(z) / 2+t^{2} f^{\prime}(z) / 2\right\} \partial_{v}, \quad X_{12}=\bar{X}_{11},
\end{aligned}
$$

where the bars mean complex conjugation. All functions are arbitrary and smooth. No nontrivial contact symmetries exist.

\section{Operators of invariant differentiation and second-order differential invariants}

For the group foliation $[14,18,23]$ of the system (3.18)-(3.23) we choose the infinite dimensional Lie subgroup generated by $X_{11}$ and $\bar{X}_{11}$ since it contains maximum number of arbitrary functions and hence maximum number of constraints for differential invariants with respect to this subgroup.

In group foliation, an important role is played by operators of invariant differentiation which, by definition, commute with any prolongation of symmetry generators $X_{11}$ and $\bar{X}_{11}$. The number of independent operators of invariant differentiation is the same as the number of independent variables, that is, five in our case. The equations determining such operators one can find in Ovsiannikov's book [20] or in our paper [14]. Here we present only the result for solving these equations which fixes the following form of operators of invariant differentiation:

$$
\begin{array}{ll}
\delta=D_{t}, \quad \Delta^{q}=q D_{q}, & \bar{\Delta}^{q}=\bar{q} D_{\bar{q}}, \\
\Delta^{z}=q^{2}\left(2 D_{z}-v_{t q} D_{q}\right), & \bar{\Delta}^{z}=\bar{q}^{2}\left(2 D_{\bar{z}}-v_{t \bar{q}} D_{\bar{q}}\right),
\end{array}
$$

where $D$ denotes total derivative with respect to its letter subscript. Operators (4.1), when acting on an invariant, generate again a (differential) invariant, increasing its order by one unit. Invariant differentiations may also generate differential invariants even when acting on a noninvariant quantities ('pre-invariants'). A basis of differential invariants is formed by invariants such that invariant differentiations can generate any invariant of an arbitrary order by repeated applications to basis invariants.

A zeroth-order invariant is $t$. A single first-order invariant is

$$
\omega_{1}=q v_{q}+\bar{q} v_{\bar{q}}+2 t v_{t}-4 v .
$$

The complete set of second-order independent differential invariants consists of 12 invariants. Indeed, the dimension of the $N$ th prolongation space, where $N$ is the order of the prolongation is $\nu_{N}=n+m \frac{(N+n) !}{N ! n !}$, where $n$ and $m$ are the numbers of independent and dependent variables, respectively, and $N=1,2,3, \ldots$ In our case $n=5, m=1$, so $\nu_{N}=5+\frac{(N+5) !}{N ! 5 !}$, which for $N=2$ yields $\nu_{2}=26$. The dimension of orbits $r_{N}$ is the rank of the system of $N$ th prolongations

of generators $X_{11}$ and $\bar{X}_{11}$, which is equal to the number of arbitrary functions of $z, \bar{z}$ that they contain. For $N=2$, we have arbitrary functions in the second prolongation of our two 
generators $f^{\prime \prime \prime \prime}(z), f^{\prime \prime \prime \prime \prime \prime}(z), \bar{f}^{\prime \prime \prime \prime}(\bar{z}), \bar{f}^{\prime \prime \prime \prime \prime \prime}(\bar{z})$ in addition to those which appear in the last line of (3.24), that is, $r_{2}=12$. The dimension of the space of invariants is $\operatorname{dim} \mathbb{Z}_{N}=\nu_{N}-r_{N}$ and for $N=2$ this becomes $\operatorname{dim} \mathbb{Z}_{2}=\nu_{2}-r_{2}=14$. Therefore, in addition to the two invariants of zeroth and first-order we must obtain 12 independent second-order invariants. All of them are listed below:

$$
\begin{aligned}
& \omega_{2}=q \bar{q} e^{-v_{t t} / 2} \\
& \omega_{3}=\Delta^{q}\left(\omega_{1}\right)=q\left(q v_{q q}+\bar{q} v_{q \bar{q}}+2 t v_{t q}-3 v_{q}\right), \\
& \bar{\omega}_{3}=\bar{\Delta}^{q}\left(\omega_{1}\right)=\bar{q}\left(\bar{q} v_{\bar{q} \bar{q}}+q v_{q \bar{q}}+2 t v_{t \bar{q}}-3 v_{\bar{q}}\right), \\
& \omega_{4}=\delta\left(\omega_{1}\right)=q v_{t q}+\bar{q} v_{t \bar{q}}+2 t v_{t t}-2 v_{t}, \\
& \omega_{5}=q \bar{q} v_{q \bar{q}}=\bar{\Delta}^{q}\left(q v_{q}\right)=\Delta^{q}\left(\bar{q} v_{\bar{q}}\right), \\
& \omega_{6}=q^{2} \bar{q}\left(2 v_{\bar{q} z}-v_{q \bar{q}} v_{t q}\right), \quad \bar{\omega}_{6}=q \bar{q}^{2}\left(2 v_{q \bar{z}}-v_{q \bar{q}} v_{t \bar{q}}\right), \\
& \omega_{7}=q^{2}\left(v_{t z}+v_{q q}-v_{t q}^{2} / 4\right), \quad \bar{\omega}_{7}=\bar{q}^{2}\left(v_{t \bar{z}}+v_{\bar{q} \bar{q}}-v_{t \bar{q}}^{2} / 4\right), \\
& \omega_{8}=q^{3} \bar{q}^{3}\left(v_{q \bar{q}} v_{z \bar{z}}-v_{q \bar{z}} v_{\bar{q} z}\right), \\
& \omega_{9}=\Delta^{z}\left(\omega_{1}\right)=q^{2}\left\{4 t v_{t z}+2 q v_{q z}+2 \bar{q} v_{\bar{q} z}-8 v_{z}\right. \\
&\left.\quad-v_{t q}\left(q v_{q q}+\bar{q} v_{q \bar{q}}+2 t v_{t q}-3 v_{q}\right)\right\}, \\
&\left.\quad-v_{t \bar{q}}\left(\bar{q} v_{\bar{q} \bar{q}}+q v_{q \bar{q}}+2 t v_{t \bar{q}}-3 v_{\bar{q}}\right)\right\} .
\end{aligned}
$$

\section{Automorphic and resolving equations}

Here we fix the form of automorphic and resolving equations using only invariant variables. We choose a set of 5 independent invariant variables (same number as in the original system (3.18)(3.23)) and three remaining differential invariants are considered as new invariant unknowns in the automorphic system (5.2) (see explanation below (5.1)). Integrability conditions of these equations together with the original system yield resolving equations for the unknown functions $F, G$ and $\bar{G}$ in (5.2). This task is much simplified by applying our modification of the method which uses commutator algebra of the operators of invariant differentiation together with its Jacobi identities $[14,18,23]$. For any solution of resolving equations for $F, G$ and $\bar{G}$ the system (5.2) possesses the automorphic property: any solution can be obtained from any other solution by a symmetry group transformation generated by $X_{11}$ and $\bar{X}_{11}$.

All our equations (3.18)-(3.23) can be expressed solely in terms of differential invariants as follows:

$$
\begin{aligned}
& \omega_{5}=2 \omega_{2} \quad(3.18), \quad \omega_{7}=0 \quad(3.19), \quad \bar{\omega}_{7}=0 \quad(3.20), \quad \omega_{6}=0 \quad(3.21), \\
& \bar{\omega}_{6}=0 \quad(3.22), \quad \omega_{8}=-2 \omega_{2}^{3} \quad(3.23) .
\end{aligned}
$$

Hence out of the twelve second-order invariants (4.2)-(4.11) there are only six independent ones. Therefore, for the second-order of prolongation we have only eight independent invariants together with $t$ and $\omega_{1}$ on the solution manifold of our system of six equations. For the group foliation, we should separate them into two groups: independent and dependent invariant variables. In order not to loose any solutions of our original equations, the number of independent invariant variables should be five, the same as in the original equations. We choose them to be $\left(t, \omega_{1}, \omega_{2}, \omega_{3}, \bar{\omega}_{3}\right)$. Thus, only three remaining invariants should be chosen as new invariant unknown functions of the five independent invariant variables

$$
\begin{aligned}
& \omega_{4}=F\left(t, \omega_{1}, \omega_{2}, \omega_{3}, \bar{\omega}_{3}\right)=\delta\left(\omega_{1}\right), \quad \omega_{9}=G\left(t, \omega_{1}, \omega_{2}, \omega_{3}, \bar{\omega}_{3}\right)=\Delta^{z}\left(\omega_{1}\right), \\
& \bar{\omega}_{9}=\bar{G}\left(t, \omega_{1}, \omega_{2}, \omega_{3}, \bar{\omega}_{3}\right)=\bar{\Delta}^{z}\left(\omega_{1}\right),
\end{aligned}
$$


which is the general form of the automorphic system. Some part of resolving equations we obtain from inner integrability conditions for these three equations together with the equations that follow from the definitions of $\omega_{3}, \bar{\omega}_{3}$

$$
\omega_{3}=\Delta^{q}\left(\omega_{1}\right), \quad \bar{\omega}_{3}=\bar{\Delta}^{q}\left(\omega_{1}\right) .
$$

These conditions are obtained by invariant cross differentiation of equations (5.2) and (5.3) where we will use the commutator algebra of operators of invariant differentiation

$$
\begin{aligned}
& {\left[\delta, \Delta^{q}\right]=\left[\delta, \bar{\Delta}^{q}\right]=0, \quad\left[\delta, \Delta^{z}\right]=-\omega_{t z} \Delta^{q}, \quad\left[\delta, \bar{\Delta}^{z}\right]=-\bar{\omega}_{t z} \bar{\Delta}^{q},} \\
& {\left[\Delta^{q}, \Delta^{z}\right]=2 \Delta^{z}-\omega_{q z} \Delta^{q}, \quad\left[\bar{\Delta}^{q}, \bar{\Delta}^{z}\right]=2 \bar{\Delta}^{z}-\bar{\omega}_{q z} \bar{\Delta}^{q},} \\
& {\left[\Delta^{q}, \bar{\Delta}^{q}\right]=0, \quad\left[\Delta^{q}, \bar{\Delta}^{z}\right]=\omega_{2} \omega_{t t} \bar{\Delta}^{q}, \quad\left[\bar{\Delta}^{q}, \Delta^{z}\right]=\omega_{2} \omega_{t t} \Delta^{q},} \\
& {\left[\Delta^{z}, \bar{\Delta}^{z}\right]=2 \omega_{2}\left(\bar{\omega}_{t z} \Delta^{q}-\omega_{t z} \bar{\Delta}^{q}\right) .}
\end{aligned}
$$

Here coefficients are the following third-order invariants

$$
\begin{aligned}
\omega_{t t} & =v_{t t t}=\delta\left(v_{t t}\right), \quad \omega_{t z}=q v_{t t q}=\Delta^{q}\left(v_{t t}\right), \quad \bar{\omega}_{t z}=\bar{q} v_{t t \bar{q}}=\bar{\Delta}^{q}\left(v_{t t}\right), \\
\omega_{q z} & =q^{2} v_{t q q}-q v_{t q}=\Delta^{q}\left(q v_{t q}\right)-2 q v_{t q} \\
& =-\left\{q^{2}\left(v_{t t z}-v_{t q} v_{t t q} / 2\right)+q v_{t q}\right\}=-\frac{1}{2} \Delta^{z}\left(v_{t t}\right)-q v_{t q}, \\
\bar{\omega}_{q z} & =\bar{q}^{2} v_{t \bar{q} \bar{q}}-\bar{q} v_{t \bar{q}}=\bar{\Delta}^{q}\left(\bar{q} v_{t \bar{q}}\right)-2 \bar{q} v_{t \bar{q}} \\
& =-\left\{\bar{q}^{2}\left(v_{t t \bar{z}}-v_{t \bar{q}} v_{t t \bar{q}} / 2\right)+\bar{q} v_{t \bar{q}}\right\}=-\frac{1}{2} \bar{\Delta}^{z}\left(v_{t t}\right)-\bar{q} v_{t \bar{q}},
\end{aligned}
$$

where the alternative expressions for $\omega_{q z}$ and $\bar{\omega}_{q z}$ in the second lines of (5.6) and (5.7) are obtained by using equations (3.19) and (3.20), respectively.

In the integrability conditions of equations (5.2) and (5.3) new third-order invariants appear which are obtained by the action of operators of invariant differentiation on second-order invariants $\omega_{2}, \omega_{3}, \bar{\omega}_{3}$. We introduce for them the following notation

$$
\omega_{i t}=\delta\left(\omega_{i}\right), \quad \omega_{i q}=\Delta^{q}\left(\omega_{i}\right), \quad \omega_{i \bar{q}}=\bar{\Delta}^{q}\left(\omega_{i}\right), \quad \omega_{i z}=\Delta^{z}\left(\omega_{i}\right), \quad \omega_{i \bar{z}}=\bar{\Delta}^{z}\left(\omega_{i}\right),
$$

where $i=2,3$, together with complex conjugates to the equations at $i=3$.

Equations (5.8) determine projections of operators of invariant differentiation on the space of invariants

$$
\begin{aligned}
& \delta=\partial_{t}+F \partial_{\omega_{1}}+\omega_{2 t} \partial_{\omega_{2}}+\omega_{3 t} \partial_{\omega_{3}}+\bar{\omega}_{3 t} \partial_{\bar{\omega}_{3}}, \\
& \Delta^{q}=\omega_{3} \partial_{\omega_{1}}+\omega_{2 q} \partial_{\omega_{2}}+\omega_{3 q} \partial_{\omega_{3}}+\bar{\omega}_{3 \bar{q}} \partial_{\bar{\omega}_{3}}, \\
& \bar{\Delta}^{q}=\bar{\omega}_{3} \partial_{\omega_{1}}+\bar{\omega}_{2 q} \partial_{\omega_{2}}+\omega_{3 \bar{q}} \partial_{\omega_{3}}+\bar{\omega}_{3 q} \partial_{\bar{\omega}_{3}}, \\
& \Delta^{z}=G \partial_{\omega_{1}}+\omega_{2 z} \partial_{\omega_{2}}+\omega_{3 z} \partial_{\omega_{3}}+\bar{\omega}_{3 \bar{z}} \partial_{\bar{\omega}_{3}}, \\
& \bar{\Delta}^{z}=\bar{G} \partial_{\omega_{1}}+\bar{\omega}_{2 z} \partial_{\omega_{2}}+\omega_{3 \bar{z}} \partial_{\omega_{3}}+\bar{\omega}_{3 z} \partial_{\bar{\omega}_{3} .} .
\end{aligned}
$$

Then invariant integrability conditions for equations (5.2) and (5.3) can be obtained by applying commutator relations between operators of invariant differentiation (5.4) to the firstorder invariant $\omega_{1}$

$$
\begin{aligned}
& \Delta^{q}(F)=\omega_{3 t}, \quad \bar{\Delta}^{q}(F)=\bar{\omega}_{3 t}, \quad \Delta^{z}(F)=\delta(G)+\omega_{3} \omega_{t z}, \quad \bar{\Delta}^{z}(F)=\delta(\bar{G})+\bar{\omega}_{3} \bar{\omega}_{t z}, \\
& \Delta^{q}(G)=2 G+\omega_{3 z}-\omega_{3} \omega_{q z}, \quad \bar{\Delta}^{q}(\bar{G})=2 \bar{G}+\bar{\omega}_{3 z}-\bar{\omega}_{3} \bar{\omega}_{q z}, \quad \bar{\Delta}^{q}(G)=\bar{\omega}_{3 \bar{z}}-\omega_{3} \omega_{q \bar{z}}, \\
& \Delta^{q}(\bar{G})=\omega_{3 \bar{z}}-\bar{\omega}_{3} \omega_{q \bar{z}}, \quad \bar{\Delta}^{z}(G)=\Delta^{z}(\bar{G})+2 \omega_{2}\left(\bar{\omega}_{3} \omega_{t z}-\omega_{3} \bar{\omega}_{t z}\right)
\end{aligned}
$$

with the reality conditions $\bar{\omega}_{3 \bar{q}}=\omega_{3 \bar{q}}$ and $\bar{\omega}_{q \bar{z}}=\omega_{q \bar{z}}$. 
These equations have already the form of resolving equations if we consider here third-order invariants defined in (5.8) as auxiliary unknowns which are functions of $t, \omega_{1}, \omega_{2}, \omega_{3}, \bar{\omega}_{3}$. Then we must add new resolving equations following from their definitions (5.8). Some of them we obtain by applying commutator relations (5.4) between invariant differentiations to independent second-order invariant variables $\omega_{2}, \omega_{3}, \bar{\omega}_{3}$ as follows

$$
\begin{aligned}
& \Delta^{q}\left(\omega_{i t}\right)=\delta\left(\omega_{i q}\right), \quad \bar{\Delta}^{q}\left(\omega_{i t}\right)=\delta\left(\bar{\omega}_{i q}\right), \quad \bar{\Delta}^{q}\left(\omega_{i q}\right)=\Delta^{q}\left(\bar{\omega}_{i q}\right), \\
& \Delta^{z}\left(\omega_{i t}\right)=\delta\left(\omega_{i z}\right)-\omega_{t z} \omega_{i q}, \quad \bar{\Delta}^{z}\left(\bar{\omega}_{i t}\right)=\delta\left(\bar{\omega}_{i z}\right)-\bar{\omega}_{t z} \bar{\omega}_{i q}, \\
& \Delta^{q}\left(\omega_{i z}\right)=\Delta^{z}\left(\omega_{i q}\right)+2 \omega_{i z}-\omega_{q z} \omega_{i q}, \quad \bar{\Delta}^{q}\left(\bar{\omega}_{i z}\right)=\bar{\Delta}^{z}\left(\bar{\omega}_{i q}\right)+2 \bar{\omega}_{i z}-\bar{\omega}_{q z} \bar{\omega}_{i q}, \\
& \Delta^{q}\left(\bar{\omega}_{i z}\right)=\bar{\Delta}^{z}\left(\omega_{i q}\right)-\omega_{q \bar{z}} \bar{\omega}_{i q}, \quad \bar{\Delta}^{q}\left(\omega_{i z}\right)=\Delta^{z}\left(\bar{\omega}_{i q}\right)-\omega_{q \bar{z}} \omega_{i q}, \\
& \Delta^{z}\left(\bar{\omega}_{i z}\right)=\bar{\Delta}^{z}\left(\omega_{i z}\right)+2 \omega_{2}\left(\bar{\omega}_{t z} \omega_{2 q}-\omega_{t z} \bar{\omega}_{2 q}\right),
\end{aligned}
$$

where $i=2,3$, plus complex conjugate equations at $i=3$. Here $\bar{\omega}_{2 t}=\omega_{2 t}$.

To obtain further resolving equations, we consider Jacobi identities between triples of operators of invariant differentiation using commutator relations (5.4)

$$
\begin{aligned}
& \Delta^{q}\left(\omega_{t z}\right)=\delta\left(\omega_{q z}\right)+2 \omega_{t z}, \quad \bar{\Delta}^{q}\left(\bar{\omega}_{t z}\right)=\delta\left(\bar{\omega}_{q z}\right)+2 \bar{\omega}_{t z}, \\
& \bar{\Delta}^{q}\left(\omega_{t z}\right)=\Delta^{q}\left(\bar{\omega}_{t z}\right)=-\omega_{2} \delta\left(\omega_{t t}\right)-\omega_{2 t} \omega_{t t}, \\
& \Delta^{z}\left(\bar{\omega}_{t z}\right)=2 \omega_{2} \delta\left(\omega_{t z}\right)+\omega_{t z}\left(2 \omega_{2 t}-\omega_{2} \omega_{t t}\right), \\
& \bar{\Delta}^{z}\left(\omega_{t z}\right)=2 \omega_{2} \delta\left(\bar{\omega}_{t z}\right)+\bar{\omega}_{t z}\left(2 \omega_{2 t}-\omega_{2} \omega_{t t}\right), \\
& \Delta^{q}\left(\bar{\omega}_{q z}\right)=-\omega_{2} \bar{\Delta}^{q}\left(\omega_{t t}\right)+\omega_{t t}\left(2 \omega_{2}-\bar{\omega}_{2 q}\right), \\
& \bar{\Delta}^{q}\left(\omega_{q z}\right)=-\omega_{2} \Delta^{q}\left(\omega_{t t}\right)+\omega_{t t}\left(2 \omega_{2}-\omega_{2 q}\right), \\
& \Delta^{z}\left(\bar{\omega}_{q z}\right)=2 \omega_{2} \bar{\Delta}^{q}\left(\omega_{t z}\right)-2 \omega_{t z}\left(2 \omega_{2}-\bar{\omega}_{2 q}\right)+\omega_{2}^{2} \omega_{t t}^{2}, \\
& \bar{\Delta}^{z}\left(\omega_{q z}\right)=2 \omega_{2} \Delta^{q}\left(\bar{\omega}_{t z}\right)-2 \bar{\omega}_{t z}\left(2 \omega_{2}-\omega_{2 q}\right)+\omega_{2}^{2} \omega_{t t}^{2}, \\
& \omega_{2}\left\{\Delta^{z}\left(\omega_{t t}\right)+2 \Delta^{q}\left(\omega_{t z}\right)\right\}=-\omega_{t t}\left(\omega_{2 z}+\omega_{2} \omega_{q z}\right)-2 \omega_{t z}\left(2 \omega_{2}+\omega_{2 q}\right), \\
& \omega_{2}\left\{\bar{\Delta}^{z}\left(\omega_{t t}\right)+2 \bar{\Delta}^{q}\left(\bar{\omega}_{t z}\right)\right\}=-\omega_{t t}\left(\bar{\omega}_{2 z}+\omega_{2} \bar{\omega}_{q z}\right)-2 \bar{\omega}_{t z}\left(2 \omega_{2}+\bar{\omega}_{2 q}\right) .
\end{aligned}
$$

For example, the first equation in (5.11) is obtained from the Jacobi identity $\left[\left[\delta, \Delta^{q}\right], \Delta^{z}\right]+$ $\left[\left[\Delta^{q}, \Delta^{z}\right], \delta\right]+\left[\left[\Delta^{z}, \delta\right], \Delta^{q}\right]=0$, while the third and fourth equations are obtained from the single Jacobi identity $\left[\left[\delta, \Delta^{z}\right], \bar{\Delta}^{z}\right]+\left[\left[\Delta^{z}, \bar{\Delta}^{z}\right], \delta\right]+\left[\left[\bar{\Delta}^{z}, \delta\right], \Delta^{z}\right]=0$.

Still more resolving equations follow from the definitions (5.5)-(5.7) of the third-order invariants that appear as coefficients of commutator algebra of invariant differentiations. Obvious consequences are obtained by invariant cross differentiations of each pair of the three equations in $(5.5)$

$$
\Delta^{q}\left(\omega_{t t}\right)=\delta\left(\omega_{t z}\right), \quad \bar{\Delta}^{q}\left(\omega_{t t}\right)=\delta\left(\bar{\omega}_{t z}\right), \quad \bar{\Delta}^{q}\left(\omega_{t z}\right)=\Delta^{q}\left(\bar{\omega}_{t z}\right) .
$$

Invariant cross differentiation of the first equation in (5.5) and (5.6), (5.7) written in the form

$$
\Delta^{z}\left(v_{t t}\right)=-2 \omega_{q z}-2 q v_{t q}, \quad \bar{\Delta}^{z}\left(v_{t t}\right)=-2 \bar{\omega}_{q z}-2 \bar{q} v_{t \bar{q}}
$$

yields

$$
\Delta^{z}\left(\omega_{t t}\right)=-2 \delta\left(\omega_{q z}\right)+\omega_{t z}^{2}-2 \omega_{t z}, \quad \bar{\Delta}^{z}\left(\omega_{t t}\right)=-2 \delta\left(\bar{\omega}_{q z}\right)+\bar{\omega}_{t z}^{2}-2 \bar{\omega}_{t z} .
$$

Invariant cross differentiations of the second and third equations in (5.5) together with (5.6) and (5.7), respectively, set in the form $\delta\left(q v_{t q}\right)=\omega_{t z}$ and $\Delta^{q}\left(q v_{t q}\right)=\omega_{q z}+2 q v_{t q}$, together with complex conjugate equations reproduce first two equations in (5.11).

Invariant cross differentiations of the second and third equations in (5.5) together with equations (5.6) and (5.7), respectively, taken in the form (5.13), yield

$$
\Delta^{z}\left(\omega_{t z}\right)=-2 \Delta^{q}\left(\omega_{q z}\right)+\omega_{q z}\left(\omega_{t z}+2\right), \quad \bar{\Delta}^{z}\left(\bar{\omega}_{t z}\right)=-2 \bar{\Delta}^{q}\left(\bar{\omega}_{q z}\right)+\bar{\omega}_{q z}\left(\bar{\omega}_{t z}+2\right) .
$$


Invariant cross differentiations of the second equation in (5.5) and equation (5.7), taken in the form (5.13), and also of the third equation in (5.5) together with (5.6) in the form (5.13) yield

$$
\begin{aligned}
& \bar{\Delta}^{z}\left(\omega_{t z}\right)=-2 \Delta^{q}\left(\bar{\omega}_{q z}\right)-4 \omega_{2 t}-\omega_{2} \omega_{t t} \omega_{t z}, \\
& \Delta^{z}\left(\bar{\omega}_{t z}\right)=-2 \bar{\Delta}^{q}\left(\omega_{q z}\right)-4 \omega_{2 t}-\omega_{2} \omega_{t t} \bar{\omega}_{t z} .
\end{aligned}
$$

Finally, the invariant cross differentiation of equations (5.6) and (5.7) taken in the form (5.13) yields the last resolving equation

$$
\bar{\Delta}^{z}\left(\omega_{q z}\right)=\Delta^{z}\left(\bar{\omega}_{q z}\right)+2 \omega_{2}\left(\omega_{t z}-\bar{\omega}_{t z}\right) .
$$

Thus, the complete set of resolving equations consists of equations (5.9), (5.10), (5.11), (5.12), (5.14), (5.15), (5.16) and (5.17).

\section{Some solutions of the extended system}

In this section we replace the task of solving the set of resolving equations by a simpler problem of finding particular solutions for the commutator algebra of operators of invariant differentiation [14, 18, 23]. As a result, we find some solutions of the Boyer-Finley equation which look very nontrivial and seem to be new. They also satisfy all other equations (3.18)(3.23) of the extended system and therefore they also solve the Legendre-transformed CMA equation (3.12). In the next section, we obtain a noninvariant solution of CMA by applying an inverse Legendre transformation to one of the obtained solutions.

The extended system (3.18)-(3.23) contains, besides Boyer-Finley equation (3.18) (from now on denoted as BF), the transformed Monge-Ampère equation which is a consequence of this system. Therefore, solutions of this system satisfy simultaneously BF and the transformed CMA and hence provide a lift of solutions of (3.18) to those of CMA.

With our choice of the symmetry for the group foliation, non-invariant solutions of the BoyerFinley equation, obtained in [3, 14] and used in [17] to generate heavenly metrics, cannot be lifted to noninvariant solutions of CMA.

Therefore, in order to solve the resolving equations of the group foliation, constructed for the extended system, we have to discover some other solutions of the BF equation (3.18), which by construction will be compatible with all other equations of our system (3.18)-(3.23). However, we have too many resolving equations to solve. Because of that, we use instead the strategy applied in our paper [14] to the single BF equation, namely, to consider the commutator algebra (5.4) of operators of invariant differentiation and make some ansatz simplifying this algebra. The most obvious ansatz is to make as many commutators as possible to vanish

$$
\begin{aligned}
& \omega_{t t}=v_{t t t}=0, \quad \omega_{t z}=0 \Rightarrow v_{t t q}=0, \quad \bar{\omega}_{t z}=0 \Rightarrow v_{t t \bar{q}}=0, \\
& \omega_{q z}=0 \Rightarrow q v_{t t z}+v_{t q}=0, \quad \bar{\omega}_{q z}=0 \Rightarrow \bar{q} v_{t t \bar{z}}+v_{t \bar{q}}=0,
\end{aligned}
$$

so that the only nonzero commutators are $\left[\Delta^{q}, \Delta^{z}\right]=2 \Delta^{z},\left[\bar{\Delta}^{q}, \bar{\Delta}^{z}\right]=2 \bar{\Delta}^{z}$. The first equation in (6.1) is very restrictive since it admits only quadratic $t$-dependence

$$
v=\frac{\alpha}{2} t^{2}+\beta t+\gamma
$$

where $\alpha, \beta, \gamma$ are functions of $q, \bar{q}, z, \bar{z}$. Plugging ansatz (6.3) in other equations of the extended system, we end up with the following solution to all of six equations of this system

$$
v=-\frac{t^{2}}{2}\left[\ln \kappa^{\prime}(z)+\ln \bar{\kappa}^{\prime}(\bar{z})\right]+t\left[\sigma(z)+q^{2} \frac{\kappa^{\prime \prime}(z)}{2 \kappa^{\prime}(z)}+\bar{\sigma}(\bar{z})+\bar{q}^{2} \frac{\bar{\kappa}^{\prime \prime}(\bar{z})}{2 \bar{\kappa}^{\prime}(\bar{z})}\right]
$$




$$
\begin{aligned}
& +2 q \bar{q} \sqrt{\kappa^{\prime}(z) \bar{\kappa}^{\prime}(\bar{z})}-\kappa(z) \bar{\kappa}(\bar{z})+\frac{q^{4}\left(3 \kappa^{\prime \prime 2}-2 \kappa^{\prime} \kappa^{\prime \prime \prime}\right)}{48 \kappa^{\prime 2}}+\frac{\bar{q}^{4}\left(3 \bar{\kappa}^{\prime \prime 2}-2 \bar{\kappa}^{\prime} \bar{\kappa}^{\prime \prime \prime}\right)}{48 \bar{\kappa}^{\prime 2}} \\
& -\frac{q^{2}}{2} \sigma^{\prime}(z)-\frac{\bar{q}^{2}}{2} \bar{\sigma}^{\prime}(\bar{z})+q \nu(z)+\bar{q} \bar{\nu}(\bar{z})+\rho(z)+\bar{\rho}(\bar{z}) .
\end{aligned}
$$

To get more general dependence on $t$, we skip the first ansatz in (6.1) and also the ansatz (6.2), since the latter does not imply the vanishing of the commutators $\left[\Delta^{q}, \Delta^{z}\right]$ and its complex conjugate. Thus, we now make the only ansatz $v_{t t q}=0$ and $v_{t t \bar{q}}=0$.

Then, without making any more assumptions, we obtain the two following solutions to the whole extended system:

$$
\begin{aligned}
v= & -(t+C)^{2}\left[\ln (t+C)-\frac{1}{2}\right]-t^{2}\left[\frac{1}{2} \ln \left(c_{1}+\bar{c}_{1}\right)-\ln a-1\right] \\
& +t\left[2 C \ln a-\frac{(d-\bar{d})^{2}}{4 a}+\varphi_{0}+\bar{\varphi}_{0}\right]+C^{2} \ln a-\frac{C(d-\bar{d})^{2}}{4 a}+\psi_{0}+\bar{\psi}_{0} \\
& +(t+C)\left\{\frac{2 \sqrt{c_{1} \bar{c}_{1}} q \bar{q}}{a}-q^{2} \frac{c_{1}}{a}+q\left[\frac{d^{\prime}}{\sqrt{c_{1}}}-\frac{\sqrt{c_{1}}(d-\bar{d})}{a}\right]-\bar{q}^{2} \frac{\bar{c}_{1}}{a}\right. \\
& \left.+\bar{q}\left[\frac{\bar{d}^{\prime}}{\sqrt{\bar{c}_{1}}}-\frac{\sqrt{\bar{c}_{1}}(d-\bar{d})}{a}\right]\right\}-\frac{q^{3}}{6} \frac{d^{\prime \prime}}{\sqrt{c_{1}}}+\frac{q^{2}}{2}\left(\frac{d^{\prime 2}}{4 c_{1}}-\frac{2 C c_{1}}{a}-\varphi_{0}^{\prime}\right) \\
& -\frac{\bar{q}^{3}}{6} \frac{\bar{d}^{\prime \prime}}{\sqrt{\bar{c}_{1}}}+\frac{\bar{q}^{2}}{2}\left(\frac{\bar{d}^{\prime 2}}{4 \bar{c}_{1}}-\frac{2 C \bar{c}_{1}}{a}-\bar{\varphi}_{0}^{\prime}\right)+q \rho_{1}+\bar{q} \bar{\rho}_{1},
\end{aligned}
$$

where $C \neq 0$ is a real constant, $a=\bar{a}=\bar{c}_{1} z+\bar{c}_{1} \bar{z}+c_{0}$ with constant $c_{1}, \bar{c}_{1}, c_{0}, d=d(z)$, $\bar{d}=\bar{d}(\bar{z}), \rho_{1}=\rho_{1}(z), \bar{\rho}_{1}=\bar{\rho}_{1}(\bar{z}), \varphi_{0}=\varphi_{0}(z), \bar{\varphi}_{0}=\bar{\varphi}_{0}(\bar{z}), \psi_{0}=\psi_{0}(z), \bar{\psi}_{0}=\bar{\psi}_{0}(\bar{z})$ are arbitrary functions and the primes denote derivatives of functions of a single variable.

The second solution obtained with the same ansatz has the form

$$
\begin{aligned}
v= & -t^{2}\left[\ln t+\frac{1}{2}\left(\ln a^{\prime}+\ln \bar{a}^{\prime}\right)-\ln (a+\bar{a})-\frac{3}{2}\right] \\
& +t\left\{2 q \bar{q} \frac{\sqrt{a^{\prime} \bar{a}^{\prime}}}{a+\bar{a}}+q^{2}\left(\frac{a^{\prime \prime}}{2 a^{\prime}}-\frac{a^{\prime}}{a+\bar{a}}\right)+\bar{q}^{2}\left(\frac{\bar{a}^{\prime \prime}}{2 \bar{a}^{\prime}}-\frac{\bar{a}^{\prime}}{a+\bar{a}}\right)\right. \\
& \left.+q\left[\frac{d^{\prime}}{\sqrt{a^{\prime}}}-\frac{\sqrt{a^{\prime}}(d-\bar{d})}{a+\bar{a}}\right]+\bar{q}\left[\frac{\bar{d}^{\prime}}{\sqrt{\bar{a}^{\prime}}}+\frac{\sqrt{\bar{a}^{\prime}}(d-\bar{d})}{a+\bar{a}}\right]-\frac{(d-\bar{d})^{2}}{4(a+\bar{a})}+\varphi_{0}+\bar{\varphi}_{0}\right\} \\
& +q^{4}\left(\frac{a^{\prime \prime 2}}{16 a^{\prime 2}}-\frac{a^{\prime \prime \prime}}{24 a^{\prime}}\right)+\frac{q^{3}}{6}\left(\frac{a^{\prime \prime} d^{\prime}}{a^{\prime 3 / 2}}-\frac{d^{\prime \prime}}{\sqrt{a^{\prime}}}\right)+q^{2}\left(\frac{d^{\prime 2}}{8 a^{\prime}}-\frac{\varphi_{0}^{\prime}}{2}\right)+q \rho_{1}+\psi_{0} \\
& +\bar{q}^{4}\left(\frac{\bar{a}^{\prime \prime 2}}{16 \bar{a}^{\prime 2}}-\frac{\bar{a}^{\prime \prime \prime}}{24 \bar{a}^{\prime}}\right)+\frac{\bar{q}^{3}}{6}\left(\frac{\bar{a}^{\prime \prime} \bar{d}^{\prime}}{\bar{a}^{\prime 3 / 2}}-\frac{\bar{d}^{\prime \prime}}{\sqrt{\bar{a}^{\prime}}}\right)+\bar{q}^{2}\left(\frac{\bar{d}^{\prime 2}}{8 \bar{a}^{\prime}}-\frac{\bar{\varphi}_{0}^{\prime}}{2}\right)+\bar{q} \bar{\rho}_{1}+\bar{\psi}_{0},
\end{aligned}
$$

where $a=a(z), \bar{a}=\bar{a}(\bar{z}), d=d(z), \bar{d}=\bar{d}(\bar{z}), \rho_{1}=\rho_{1}(z), \bar{\rho}_{1}=\bar{\rho}_{1}(\bar{z}), \varphi_{0}=\varphi_{0}(z), \bar{\varphi}_{0}=\bar{\varphi}_{0}(\bar{z})$, $\psi_{0}=\psi_{0}(z), \bar{\psi}_{0}=\bar{\psi}_{0}(\bar{z})$ are all arbitrary functions of a single variable. It is interesting to note that the functions $a$ and $\bar{a}$ come from the general solution

$$
\varepsilon(z, \bar{z})=\frac{\sqrt{a^{\prime}(z) \bar{a}^{\prime}(\bar{z})}}{a+\bar{a}}
$$

of the Liouville equation $\left(\ln \varepsilon^{2}\right)_{z \bar{z}}=2 \varepsilon^{2}$.

We note that in the process we have obtained some solutions (6.4), (6.5), and (6.6) of the Boyer-Finley equation in the form (3.18), which seem to be new.

We also mention separable solutions of the Boyer-Finley equation, which reduce in essence to solutions of the Liouville equation, obtained by Tod [26] and recently by Dunajski et al. [5]. 
These solutions turn out to be invariant with respect to one-parameter subgroup of the conformal symmetry group of the Boyer-Finley equation, so it generates a metric with at least two Killing vectors, one of which comes from the BF equation, being itself a rotational symmetry reduction of CMA. We see that the Liouville equation arises also in the present paper but in a different context, not implying separability of our solutions.

\section{Simultaneous solutions to Boyer-Finley and complex Monge-Ampère equations}

We study here the most nontrivial solution (6.6) of the extended system. It should be transformed to a simultaneous solution $u$ of the Legendre-transformed CMA (3.12) and BoyerFinley (3.9) equations together with other equations (3.10) and (3.11) of the extended system. To achieve this, we apply to solution (6.6) one-dimensional Legendre transformation contained in formula (3.15): $\rho=-w_{t}, u=w-t w_{t}$ together with $w=v_{t}$ to obtain

$$
t=\frac{(a+\bar{a})}{\sqrt{a^{\prime} \bar{a}^{\prime}}} e^{\rho / 2}
$$

and the solution becomes

$$
\begin{aligned}
u= & \frac{2(a+\bar{a})}{\sqrt{a^{\prime} \bar{a}^{\prime}}} e^{\rho / 2}+2 q \bar{q} \frac{\sqrt{a^{\prime} \bar{a}^{\prime}}}{(a+\bar{a})}+q^{2}\left(\frac{a^{\prime \prime}}{2 a^{\prime}}-\frac{a^{\prime}}{a+\bar{a}}\right)+\bar{q}^{2}\left(\frac{\bar{a}^{\prime \prime}}{2 \bar{a}^{\prime}}-\frac{\bar{a}^{\prime}}{a+\bar{a}}\right) \\
& +q\left[\frac{d^{\prime}}{\sqrt{a^{\prime}}}-\frac{\sqrt{a^{\prime}}(d-\bar{d})}{a+\bar{a}}\right]+\bar{q}\left[\frac{\bar{d}^{\prime}}{\sqrt{\bar{a}^{\prime}}}+\frac{\sqrt{\bar{a}^{\prime}}(d-\bar{d})}{a+\bar{a}}\right]-\frac{(d-\bar{d})^{2}}{4(a+\bar{a})}+\varphi_{0}+\bar{\varphi}_{0},
\end{aligned}
$$

where we change the notation of independent variables from $z, \bar{z}$ to $\sigma, \bar{\sigma}$ for the functions $a$, $\bar{a}, d, \bar{d}, \varphi_{0}, \bar{\varphi}_{0}$. The expression (7.1) is a solution of the Legendre-transformed CMA (3.12). To transform back (7.1) to a solution of the CMA equation (3.13), which after changing the notation $v \mapsto \Omega$ and setting $s=\sigma e^{\rho / 4}, \bar{s}=\bar{\sigma} e^{\rho / 4}$ becomes

$$
\Omega_{p \bar{p}} \Omega_{\sigma \bar{\sigma}}-\Omega_{p \bar{\sigma}} \Omega_{\sigma \bar{p}}=e^{\rho / 2}
$$

we apply to solution (7.1) the inverse Legendre transformation of the form (3.14)

$$
\Omega=u-q u_{q}-\bar{q} u_{\bar{q}}, \quad p=-u_{q}, \quad \bar{p}=-u_{\bar{q}} .
$$

To eliminate $q, \bar{q}$ from the solution, the two latter equations in (7.3) can easily be solved for $q$ and $\bar{q}$

$$
q=\alpha p+\beta \bar{p}+\gamma, \quad \bar{q}=\bar{\alpha} \bar{p}+\beta p+\bar{\gamma},
$$

where

$$
\begin{aligned}
& \alpha=\frac{A}{\Delta}, \quad \bar{\alpha}=\frac{\bar{A}}{\Delta}, \quad \beta=\bar{\beta}=\frac{B}{\Delta}, \quad \gamma=\frac{C}{\Delta}, \quad \bar{\gamma}=\frac{\bar{C}}{\Delta}, \\
& A=\bar{a}^{\prime}\left[2 a^{\prime 2}-a^{\prime \prime}(a+\bar{a})\right], \quad \bar{A}=a^{\prime}\left[2 \bar{a}^{\prime 2}-\bar{a}^{\prime \prime}(a+\bar{a})\right], \\
& B=\bar{B}=2\left(a^{\prime} \bar{a}^{\prime}\right)^{3 / 2}, \quad C=\frac{1}{\sqrt{a^{\prime}}}\left(d^{\prime} \bar{A}+a^{\prime} \bar{D}\right), \quad \bar{C}=\frac{1}{\sqrt{\bar{a}^{\prime}}}\left(\bar{d}^{\prime} A+\bar{a}^{\prime} D\right), \\
& D=\bar{a}^{\prime}\left[2 a^{\prime} d^{\prime}-a^{\prime \prime}(d-\bar{d})\right], \quad \bar{D}=a^{\prime}\left[2 \bar{a}^{\prime} \bar{d}^{\prime}+\bar{a}^{\prime \prime}(d-\bar{d})\right] \\
& \Delta=a^{\prime \prime} \bar{a}^{\prime \prime}(a+\bar{a})-2 a^{\prime \prime} \bar{a}^{\prime 2}-2 \bar{a}^{\prime \prime} a^{\prime 2} .
\end{aligned}
$$


Then according to the formula (7.3) for $\Omega$, solution (7.1) finally becomes

$$
\begin{aligned}
\Omega= & \frac{\bar{\alpha}}{2} p^{2}+\frac{\alpha}{2} \bar{p}^{2}+\beta p \bar{p}+\gamma p+\bar{\gamma} \bar{p}+\frac{\Delta\left(\alpha \gamma^{2}+\bar{\alpha} \bar{\gamma}^{2}-2 \beta \gamma \bar{\gamma}\right)}{2 a^{\prime} \bar{a}^{\prime}(a+\bar{a})} \\
& -\frac{(d-\bar{d})^{2}}{4(a+\bar{a})}+\frac{2(a+\bar{a})}{\sqrt{a^{\prime} \bar{a}^{\prime}}} e^{\rho / 2}+\varphi_{0}+\bar{\varphi}_{0} .
\end{aligned}
$$

\section{Anti-self-dual Ricci-flat metric of Euclidean signature}

It is well known [21] that solutions of the CMA equation (3.13) govern the Kähler metric

$$
d s^{2}=2\left(v_{p \bar{p}} d p d \bar{p}+v_{p \bar{s}} d p d \bar{s}+v_{s \bar{p}} d s d \bar{p}+v_{s \bar{s}} d s d \bar{s}\right)
$$

which is anti-self-dual (ASD) Einstein vacuum (Ricci-flat) metric with Euclidean signature. The transformation $s=\sigma e^{\rho / 4}, \bar{s}=\bar{\sigma} e^{\rho / 4}$, which maps equation (3.13) into equation (7.2), does not change the metric (8.1) and hence the solution (7.5) of the equation (7.2) can be used as a potential of the Kähler metric

$$
d s^{2}=2\left(\Omega_{p \bar{p}} d p d \bar{p}+\Omega_{p \bar{\sigma}} d p d \bar{\sigma}+\Omega_{\sigma \bar{p}} d \sigma d \bar{p}+\Omega_{\sigma \bar{\sigma}} d \sigma d \bar{\sigma}\right) .
$$

Plugging our solution (7.5) into the metric (8.2) we obtain an explicit Einstein vacuum metric with Euclidean signature

$$
\begin{aligned}
d s^{2}= & 2 \beta d p d \bar{p}+2\left(p \bar{\alpha}_{\bar{\sigma}}+\bar{p} \beta_{\bar{\sigma}}+\gamma_{\bar{\sigma}}\right) d p d \bar{\sigma}+2\left(\bar{p} \alpha_{\sigma}+p \beta_{\sigma}+\bar{\gamma}_{\sigma}\right) d \bar{p} d \sigma \\
& +\left\{p^{2} \bar{\alpha}_{\sigma \bar{\sigma}}+\bar{p}^{2} \alpha_{\sigma \bar{\sigma}}+2 p \bar{p} \beta_{\sigma \bar{\sigma}}+2 p \gamma_{\sigma \bar{\sigma}}+2 \bar{p} \bar{\gamma}_{\sigma \bar{\sigma}}\right. \\
& \left.+\left[\frac{\Delta\left(\alpha \gamma^{2}+\bar{\alpha} \bar{\gamma}^{2}-2 \beta \gamma \bar{\gamma}\right)}{a^{\prime} \bar{a}^{\prime}(a+\bar{a})}-\frac{(d-\bar{d})^{2}}{2(a+\bar{a})}\right]_{\sigma \bar{\sigma}}-\frac{2}{\beta} e^{\rho / 2}\right\} d \sigma d \bar{\sigma} .
\end{aligned}
$$

From the definitions (7.4) of the metric coefficients in (8.3), we see that the only singularities of the metric in a bounded domain are zeros of the denominator

$$
\Delta=a^{\prime \prime} \bar{a}^{\prime \prime}(a+\bar{a})-2 a^{\prime \prime} \bar{a}^{\prime 2}-2 \bar{a}^{\prime \prime} a^{\prime 2}=0 .
$$

Indeed, the existence condition for our solution is $a^{\prime}(\sigma) \cdot \bar{a}^{\prime}(\bar{\sigma}) \neq 0$, so that $a$ and $\bar{a}$ are not constant and hence $a(\sigma)+\bar{a}(\bar{\sigma}) \neq 0$. The general solution for the singularity condition (8.4) in the case of $a^{\prime \prime} \cdot \bar{a}^{\prime \prime} \neq 0$ is

$$
a=i \lambda-\frac{1}{a_{1} \sigma+a_{0}}, \quad \bar{a}=-i \lambda-\frac{1}{\bar{a}_{1} \bar{\sigma}+\bar{a}_{0}}
$$

and if $a^{\prime \prime}=\bar{a}^{\prime \prime}=0$, we have

$$
a=a_{1} \sigma+a_{0}, \quad \bar{a}=\bar{a}_{1} \bar{\sigma}+\bar{a}_{0} .
$$

Here $\lambda$ and $a_{0}, a_{1}$ are real and complex constants, respectively. Thus, avoiding the choices (8.5) and (8.6) for $a$ and $\bar{a}$, we have the metric (8.3) free of singularities in a bounded domain.

To compute Riemann curvature two-forms, we choose the tetrad coframe [7] to be

$$
e^{1}=\frac{1}{\Omega_{p \bar{p}}}\left(\Omega_{p \bar{p}} d p+\Omega_{z \bar{p}} d z\right), \quad e^{2}=\Omega_{p \bar{p}} d \bar{p}+\Omega_{p \bar{z}} d \bar{z} \quad e^{3}=\frac{e^{\rho / 2}}{\Omega_{p \bar{p}}} d z, \quad e^{4}=d \bar{z},
$$

so that the metric (8.2) with the aid of equation (7.2) takes the form $d s^{2}=2\left(e^{1} e^{2}+e^{3} e^{4}\right)$, where we plug in the solution (7.5) for $\Omega$. 
Using the computer algebra package EXCALC under REDUCE [22], we calculated Riemannian curvature two-forms [7] for the metric (8.3)

$$
\begin{aligned}
& R_{1}^{1}=2 e^{-\rho / 2} \frac{\left|a^{\prime}\right|^{5}}{\Delta^{3}}\left|2 a^{\prime \prime \prime} a^{\prime}-3\left(a^{\prime \prime}\right)^{2}\right|^{2}\left(e^{1} \wedge e^{2}-e^{3} \wedge e^{4}\right)=-R_{2}^{2}=-R_{3}^{3}=R_{4}^{4}, \\
& R_{2}^{1}=R_{4}^{1}=R_{1}^{2}=R_{3}^{2}=R_{2}^{3}=R_{4}^{3}=R_{1}^{4}=R_{3}^{4}=0
\end{aligned}
$$

with the remaining curvature two-forms being too lengthy for presentation here. For example

$$
\begin{aligned}
R_{3}^{1}= & \frac{\left(\bar{a}^{\prime}\right)^{3} e^{-\rho}}{\sqrt{a^{\prime}} \Delta^{3}}\left\{a^{\prime \prime}\left(4 a^{\prime \prime \prime} a^{\prime}-3 a^{\prime \prime 2}\right)\left(5 \Delta+18 a^{\prime 2} \bar{a}^{\prime \prime}\right)\right. \\
& \left.-12 a^{\prime 2} a^{\prime \prime \prime 2}\left[(a+\bar{a}) \bar{a}^{\prime \prime}-2 \bar{a}^{\prime 2}\right]+4 a^{\prime 2} a^{\prime \prime \prime \prime} \Delta\right\} e^{2} \wedge e^{3} \\
& -2 e^{-\rho / 2} \frac{a^{\prime 2} \sqrt{\bar{a}^{\prime}}}{\Delta^{3}}\left|2 a^{\prime} a^{\prime \prime \prime}-3 a^{\prime \prime 2}\right|^{2} e^{1} \wedge e^{4} .
\end{aligned}
$$

We see that the only singularities of the curvature in a bounded domain are poles at $\Delta=0$ together with $a^{\prime} \cdot \bar{a}^{\prime}=0$ which are the same as those of the metric. We note that our solution (7.5) does not exist if the singularities conditions are satisfied.

We observe that though the metric (8.3) contains $p$ and $\bar{p}$, the curvature is independent of these variables. This is due to the quadratic dependence of the metric on $p$ and $\bar{p}$. Therefore, the curvature components do not vanish outside of a bounded domain, so our metric is not of an instanton type.

One may wonder if there are choices of $a(\sigma), \bar{a}(\bar{\sigma})$ for which all components of the Riemannian tensor vanish, so that our solution describes a flat space. The inspection of our result for the Riemannian curvature two-forms (8.7), (8.8) gives the conditions for zero curvature to be of the form

$$
a^{\prime \prime \prime}=\frac{3 a^{\prime \prime 2}}{2 a^{\prime}}, \quad \bar{a}^{\prime \prime \prime}=\frac{3 \bar{a}^{\prime \prime 2}}{2 \bar{a}^{\prime}},
$$

which yield

$$
a=-\frac{4}{k(k \sigma+l)}, \quad \bar{a}=-\frac{4}{\bar{k}(\bar{k} \bar{\sigma}+\bar{l})},
$$

where $k, l, \bar{k}, \bar{l}$ are arbitrary constants. This result coincides with the singularity condition (8.5) at $\lambda=0$ for which our solution (7.5) does not exists and therefore this is not allowed, so that we will never have a flat space for any allowed choice of $a, \bar{a}$.

We have succeeded in converting the solution (7.1) into solution (7.5) of CMA equation (7.2) because of the quadratic dependence of solution (7.1) on $q$, $\bar{q}$, which allowed us to eliminate these variables in terms of $p, \bar{p}$ by solving a linear system. For a more general dependence of solutions on $q, \bar{q}$, this transition could happen to be impossible to be performed explicitly. In this case, we need to transform Kähler metric (8.1) to the one that involves a solution $u$ of the Legendre-transformed CMA (3.12) (solution (7.1) in our example) by applying the inverse Legendre transformation (3.14) with $v$ replaced by $\Omega$ to the metric (8.2). The resulting metric has the form

$$
\begin{aligned}
d s^{2}= & \frac{2}{\Delta_{-}}\left\{u_{q \bar{q}}^{2}\left(u_{q q} d q^{2}+u_{\bar{q} \bar{q}} d \bar{q}^{2}\right)+\Delta_{+} u_{q \bar{q}} d q d \bar{q}+u_{q q} u_{\bar{q} z}^{2} d z^{2}+u_{\bar{q} \bar{q}} u_{q \bar{z}}^{2} d \bar{z}^{2}\right. \\
& +\left(\Delta_{-} u_{z \bar{z}}+2 u_{q \bar{q}} u_{q \bar{z}} u_{\bar{q} z}\right) d z d \bar{z}+2 u_{q \bar{q}}\left(u_{q q} u_{\bar{q} z} d q d z+u_{\bar{q} \bar{q}} u_{q \bar{z}} d \bar{q} d \bar{z}\right) \\
& \left.+\Delta_{+}\left(u_{q \bar{z}} d q d \bar{z}+u_{\bar{q} z} d \bar{q} d z\right)\right\}
\end{aligned}
$$

where $\Delta_{-}=u_{q q} u_{\bar{q} \bar{q}}-u_{q \bar{q}}^{2}$ and $\Delta_{+}=u_{q q} u_{\bar{q} \bar{q}}+u_{q \bar{q}}^{2}$. Metric (8.9) is again anti-self-dual and Ricci-flat with Euclidean signature for any solution $u$ of the transformed CMA equation (3.12). 


\section{Invariant and noninvariant solutions}

We are interested here in ASD Ricci-flat metrics of Euclidean signature that do not admit any Killing vectors. This implies solutions of CMA equation for the Kähler potential of the metric to be noninvariant solutions of CMA. This means that we allow only solution manifolds noninvariant under point symmetries of CMA equation. For a set of solutions of the Boyer-Finley equation we presented such an analysis in detail in [14]. In our case, for CMA equation (7.2), dependent of an extra parameter $\rho$, we have the following generators of one-parameter point symmetries subgroups

$$
\begin{array}{ll}
X_{a_{1}}=a_{1}(\rho)\left(4 \partial_{\rho}+\Omega \partial_{\Omega}\right), & Y_{b}=b(\rho)\left(p \partial_{p}+\bar{p} \partial_{\bar{p}}+\Omega \partial_{\Omega}\right), \\
Z_{c_{1}}=i c_{1}(\rho)\left(\sigma \partial_{\sigma}-\bar{\sigma} \partial_{\bar{\sigma}}\right), & V_{g}=g_{p} \partial_{\sigma}-g_{\sigma} \partial_{p}, \quad \bar{V}_{\bar{g}}=\bar{g}_{\bar{p}} \partial_{\bar{\sigma}}-\bar{g}_{\bar{\sigma}} \partial_{\bar{p}}, \\
W_{h}=h \partial_{\Omega}, \quad \bar{W}_{\bar{h}}=\bar{h} \partial_{\Omega}, &
\end{array}
$$

where $g=g(p, \sigma, \rho), \bar{g}=\bar{g}(\bar{p}, \bar{\sigma}, \rho)$ and $h=h(p, \sigma, \rho), \bar{h}=\bar{h}(\bar{p}, \bar{\sigma}, \rho)$ are arbitrary holomorphic and anti-holomorphic functions of two complex and one real variable, $a_{1}(\rho), b(\rho)$, and $c_{1}(\rho)$ are arbitrary real-valued functions of a single variable. We present here the table of commutators of the symmetry generators where the value of the commutator of the generators standing at the $i$ th row and at the $j$ th column is given at the intersection of the $i$ th row and $j$ th column. The primes denote derivatives of functions of a single variable $\rho$.

Table 1. Commutators of point symmetries of parameter-dependent CMA.

\begin{tabular}{c|c|c|c|c|c|c|c}
\hline & $X_{a_{1}}$ & $Y_{b}$ & $Z_{c_{1}}$ & $V_{g}$ & $\bar{V}_{\bar{g}}$ & $W_{h}$ & $\bar{W}_{\bar{h}}$ \\
\hline$X_{a_{1}}$ & 0 & $4 Y_{a_{1} b^{\prime}}$ & $4 Z_{a_{1} c_{1}^{\prime}}$ & $4 V_{a_{1} g_{\rho}}$ & $4 \bar{V}_{a_{1} \bar{g}_{\rho}}$ & $4 W_{a_{1} h_{\rho}}$ & $4 \bar{W}_{a_{1} \bar{h}_{\rho}}$ \\
\hline$Y_{b}$ & & 0 & 0 & $V_{b\left(p g_{p}-g\right)}$ & $\bar{V}_{b\left(\bar{p} \bar{g}_{\bar{p}-\bar{g})}\right.}$ & $W_{b\left(p h_{p}-h\right)}$ & $\bar{W}_{b\left(\bar{p} \bar{h}_{\bar{p}-\bar{h})}\right.}$ \\
\hline$Z_{c_{1}}$ & & & 0 & $i V_{c_{1}\left(\sigma g_{\sigma}-g\right)}$ & $-i \bar{V}_{c_{1}\left(\bar{\sigma} \bar{g}_{\bar{\sigma}}-\bar{g}\right)}$ & $i W_{c_{1} \sigma h_{\sigma}}$ & $-i \bar{W}_{c_{1} \bar{\sigma} \bar{h}_{\bar{\sigma}}}$ \\
\hline$V_{g}$ & & & & 0 & 0 & $W_{V_{g}(h)}$ & 0 \\
\hline $\bar{V}_{\bar{g}}$ & & & & & 0 & 0 & $\bar{W}_{\bar{V}_{\bar{g}}(\bar{h})}$ \\
\hline$W_{h}$ & & & & & & 0 & 0 \\
\hline $\bar{W}_{\bar{h}}$ & & & & & & & 0 \\
\hline
\end{tabular}

For any solution $\Omega=f(p, \bar{p}, \sigma, \bar{\sigma}, \rho)$ of equation (7.2), the condition for this solution to be invariant under a generator $X$ of an arbitrary one-parameter symmetry subgroup of (7.2) has the form

$$
\left.X(f-\Omega)\right|_{\Omega=f}=0 .
$$

With $X$ equal to a linear combination of the generators (9.1) with arbitrary constant coefficients (in our case absorbed by arbitrary functions in the generators), the invariance condition (9.2) for the solution $\Omega=f(p, \bar{p}, \sigma, \bar{\sigma}, \rho)$ becomes

$$
\begin{aligned}
g_{p} f_{\sigma} & -g_{\sigma} f_{p}+\bar{g}_{\bar{p}} f_{\bar{\sigma}}-\bar{g}_{\bar{\sigma}} f_{\bar{p}}+b(\rho)\left(p f_{p}+\bar{p} f_{\bar{p}}\right)+i \tilde{c}(\rho)\left(\sigma f_{\sigma}-\bar{\sigma} f_{\bar{\sigma}}\right) \\
& +4 \tilde{a}(\rho) f_{\rho}-(\tilde{a}(\rho)+b(\rho)) f-h-\bar{h}=0 .
\end{aligned}
$$

To check the non-invariance of the solution (7.5), one should plug $f$ equal to the right-hand side of (7.5) in the condition (9.3) and determine either a contradiction or some special forms of the coefficient functions in (7.5) which should be avoided for a noninvariant solution. 
To simplify the invariance condition, we have to use optimal Lie subalgebras instead of the general one-dimensional subalgebra used in (9.3) with the generator

$$
X=X_{\tilde{a}(\rho)}+Y_{b(\rho)}+Z_{\tilde{c}(\rho)}+V_{g}+\bar{V}_{\bar{g}}+W_{h}+\bar{W}_{\bar{h}} .
$$

For this purpose, we study the adjoint group actions on one-dimensional Lie subalgebras [19]. Here we must distinguish two cases.

Case I: $\tilde{a}(\rho) \neq 0$. In this case, we use the adjoint group actions

$$
\operatorname{Ad}\left(\exp \left(-\frac{1}{4} Y_{e^{\int d \rho / \tilde{a}}}\right)\right)\left(X_{\tilde{a}}\right)=X_{\tilde{a}}-Y_{b}, \quad \operatorname{Ad}\left(\exp \left(-\frac{1}{4} Z_{e^{\int(\tilde{c} / \tilde{a}) d \rho}}\right)\right)\left(X_{\tilde{a}}\right)=X_{\tilde{a}}-Z_{\tilde{c}}
$$

to eliminate $Y_{b}$ and $Z_{\tilde{c}}$ in (9.4), so that the optimal subalgebra becomes

$$
X=X_{\tilde{a}}+V_{g}+\bar{V}_{\bar{g}}+W_{h}+\bar{W}_{\bar{h}}
$$

which results in setting $b=\tilde{c}=0$ in the invariance condition (9.3):

$$
g_{p} f_{\sigma}-g_{\sigma} f_{p}+\bar{g}_{\bar{p}} f_{\bar{\sigma}}-\bar{g}_{\bar{\sigma}} f_{\bar{p}}+\tilde{a}(\rho)\left(4 f_{\rho}-f\right)-h-\bar{h}=0 .
$$

Case II: $\tilde{a}(\rho)=0$. Then using the adjoint group actions

$$
\operatorname{Ad}\left(\exp \left(\varepsilon W_{h}\right)\left(Y_{b}\right)=Y_{b}+\varepsilon W_{b\left(p h_{p}-h\right)}, \quad \operatorname{Ad}\left(\exp \left(\varepsilon V_{g}\right)\left(Y_{b}\right)=Y_{b}+\varepsilon V_{\tilde{g}}\right.\right.
$$

together with their complex conjugates, we can eliminate $V_{g}, W_{h}, \bar{V}_{\bar{g}}, \bar{W}_{\bar{h}}$ from (9.4) at $\tilde{a}(\rho)=0$ and the second optimal one-dimensional subalgebra becomes

$$
X=Y_{b}+Z_{\tilde{c}}
$$

The invariance condition (9.3) in the Case II due to the result (9.6) for the optimal subalgebra implies $g=\bar{g}=\tilde{a}=h=\bar{h}=0$, so that the invariance condition (9.3) becomes

$$
b(\rho)\left(p f_{p}+\bar{p} f_{\bar{p}}-f\right)+i \tilde{c}(\rho)\left(\sigma f_{\sigma}-\bar{\sigma} f_{\bar{\sigma}}\right)=0 .
$$

After some routine computations we discover that in both Cases I and II our solution (7.5) generically does not satisfy the invariance conditions (9.5) and (9.7), respectively, and hence it is noninvariant in the generic case, that is, with no restrictions on arbitrary functions of one variable in solution (7.5). A full classification of particular choices of the functional parameters that correspond to invariant solutions presents a difficult problem which is still expecting its solution.

\section{Conclusion}

The problem of obtaining explicitly the metric of $K 3$ gravitational instanton or at least some pieces of it, which will not admit any Killing vectors (no continuous symmetries), has motivated our search for non-invariant solutions to the elliptic complex Monge-Ampère equation. In recent years we have developed three approaches to the latter problem: partner symmetries, that is, invariance with respect to a certain nonlocal symmetry, symmetry reduction with respect to symmetry group parameters introduced explicitly in the theory as new independent variables and a version of the group foliation method, which is based on solving commutator algebra relations for operators of invariant differentiation. In this paper, we have combined all these approaches by introducing explicitly symmetry group parameters into the extended system of six PDEs, which determine partner symmetries of CMA, performing symmetry reductions of these 
equations with respect to the group parameters and, finally, applying the group foliation to the reduced system. Since the final reduced system contains the Boyer-Finley equation together with CMA, though not in the same variables, a solution to the extended system provides a lift from some solutions of the elliptic BF equation to noninvariant solutions of CMA, that is, from rotationally invariant to noninvariant solutions of CMA.

To provide an example of our solution procedure, we have chosen the most obvious ansatzes simplifying the commutator algebra of operators of invariant differentiation and obtained some solutions to our extended system which, after Legendre transformations, became new simultaneous solutions to a parameter-dependent CMA equation and the BF equation. Using the most general of the obtained solutions, we obtained an anti-self-dual Ricci-flat Einstein-Kähler metric with Euclidean signature and computed Riemannian curvature two-forms. The only singularities of the metric and the curvature, located in a bounded domain, exist only for a very special choice of arbitrary functions of one variable in our solution and therefore they can easily be avoided. Considering in detail the conditions for our solution to be invariant under optimal symmetry subgroups of CMA, we have proved that this is a noninvariant solution in the generic case (that is, with no special restrictions on functional parameters) and hence our metric does not admit any Killing vectors.

Our main goal here was to demonstrate how our methods may yield ASD Ricci-flat metrics without Killing vectors, for which purpose we have chosen simplest possible non-invariant solutions of CMA. Therefore, it is not surprising that our ansatz for the solution was too restrictive to obtain an instanton metric, so that the curvature is not concentrated in a bounded domain. Even though noninvariant instanton solutions were not found, we believe that the groundwork for future research has been laid, so that a more systematic study of possible solutions and also different reductions of the extended system in group parameters will provide gravitational instanton metrics of Euclidean signature without Killing vectors.

\section{Acknowledgement}

We thank our referees for their encouragement and criticism which hopefully improved our paper. The research of M.B. Sheftel was supported in part by the research grant from Boğaziçi University Scientific Research Fund (BAP), research project No. 6324.

\section{References}

[1] Atiyah M.F., Hitchin N.J., Singer I.M., Self-duality in four-dimensional Riemannian geometry, Proc. Roy. Soc. London Ser. A 362 (1978), 425-461.

[2] Boyer C.P., Finley III J.D., Killing vectors in self-dual, Euclidean Einstein spaces, J. Math. Phys. 23 (1982), $1126-1130$.

[3] Calderbank D.M.J., Tod P., Einstein metrics, hypercomplex structures and the Toda field equation, Differential Geom. Appl. 14 (2001), 199-208, math.DG/9911121.

[4] Dunajski M., Solitons, instantons, and twistors, Oxford Graduate Texts in Mathematics, Vol. 19, Oxford University Press, Oxford, 2010.

[5] Dunajski M., Gutowski J., Sabra W., Enhanced Euclidean supersymmetry, 11D supergravity and SU( $\infty)$ Toda equation, J. High Energy Phys. 2013 (2013), no. 10, 089, 20 pages, arXiv:1301.1896.

[6] Dunajski M., Mason L.J., Twistor theory of hyper-Kähler metrics with hidden symmetries, J. Math. Phys. 44 (2003), 3430-3454, math.DG/0301171.

[7] Eguchi T., Gilkey P.B., Hanson A.J., Gravitation, gauge theories and differential geometry, Phys. Rep. 66 (1980), 213-393.

[8] Hitchin N., Compact four-dimensional Einstein manifolds, J. Differential Geometry 9 (1974), 435-441.

[9] Lie S., Über Differentialinvarianten, Math. Ann. 24 (1884), 537-578. 
[10] Malykh A.A., Nutku Y., Sheftel M.B., Partner symmetries of the complex Monge-Ampère equation yield hyper-Kähler metrics without continuous symmetries, J. Phys. A: Math. Gen. 36 (2003), 10023-10037, math-ph/0305037.

[11] Malykh A.A., Nutku Y., Sheftel M.B., Partner symmetries and non-invariant solutions of four-dimensional heavenly equations, J. Phys. A: Math. Gen. 37 (2004), 7527-7545, math-ph/0403020.

[12] Malykh A.A., Nutku Y., Sheftel M.B., Lift of noninvariant solutions of heavenly equations from three to four dimensions and new ultra-hyperbolic metrics, J. Phys. A: Math. Theor. 40 (2007), 9371-9386, arXiv:0704.3335.

[13] Malykh A.A., Sheftel M.B., Recursions of symmetry orbits and reduction without reduction, SIGMA 7 (2011), 043, 13 pages, arXiv:1005.0153.

[14] Martina L., Sheftel M.B., Winternitz P., Group foliation and non-invariant solutions of the heavenly equation, J. Phys. A: Math. Gen. 34 (2001), 9243-9263, math-ph/0108004.

[15] Mason L.J., Newman E.T., A connection between the Einstein and Yang-Mills equations, Comm. Math. Phys. 121 (1989), 659-668.

[16] Mason L.J., Woodhouse N.M.J., Integrability, self-duality, and twistor theory, London Mathematical Society Monographs. New Series, Vol. 15, The Clarendon Press, Oxford University Press, New York, 1996.

[17] Nutku Y., Sheftel M.B., A family of heavenly metrics, gr-qc/0105088.

[18] Nutku Y., Sheftel M.B., Differential invariants and group foliation for the complex Monge-Ampère equation, J. Phys. A: Math. Gen. 34 (2001), 137-156.

[19] Olver P.J., Applications of Lie groups to differential equations, Graduate Texts in Mathematics, Vol. 107, Springer-Verlag, New York, 1986.

[20] Ovsiannikov L.V., Group analysis of differential equations, Academic Press Inc., New York, 1982.

[21] Plebañski J.F., Some solutions of complex Einstein equations, J. Math. Phys. 16 (1975), 2395-2402.

[22] Schrüfer E., EXCALC: A differential geometry package, in REDUCE, User's and Contributed Packages Manual, Version 3.8, Editor A.C. Hearn, Santa Monica, CA, 2003, 333-343.

[23] Sheftel M.B., Method of group foliation and non-invariant solutions of partial differential equations. Example: the heavenly equation, Eur. Phys. J. B 29 (2002), 203-206.

[24] Sheftel M.B., Malykh A.A., Lift of invariant to non-invariant solutions of complex Monge-Ampère equations, J. Nonlinear Math. Phys. 15 (2008), suppl. 3, 385-395, arXiv:0802.1463.

[25] Sheftel M.B., Malykh A.A., On classification of second-order PDEs possessing partner symmetries, J. Phys. A: Math. Theor. 42 (2009), 395202, 20 pages, arXiv:0904.2909.

[26] Tod K.P., Scalar-flat Kähler and hyper-Kähler metrics from Painlevé-III, Classical Quantum Gravity 12 (1995), 1535-1547, gr-qc/0105088.

[27] Vessiot E., Sur l'intégration des systèmes différentiels qui admettent des groupes continus de transformations, Acta Math. 28 (1904), 307-349.

[28] Yau S.T., Calabi's conjecture and some new results in algebraic geometry, Proc. Nat. Acad. Sci. USA 74 (1977), 1798-1799. 